\title{
Dicke model and environment-induced entanglement in ion-cavity QED
}

\author{
K. Härkönen, ${ }^{1, *}$ F. Plastina, ${ }^{2}$ and S. Maniscalco ${ }^{1}$ \\ ${ }^{1}$ Department of Physics and Astronomy, University of Turku, FI-20014 Turku, Finland \\ ${ }^{2}$ Dip. Fisica, Università della Calabria, and INFN - Gruppo collegato di Cosenza, Arcavacata di Rende, Cosenza 87036, Italy
}

(Dated: November 2, 2018)

\begin{abstract}
We investigate realistic experimental conditions under which the collective Dicke model can be implemented in the ion-cavity QED context. We show how ideal subradiance and superradiance can be observed and we propose an experiment to generate entanglement exploiting the existence of the subradiant state. We explore the conditions to achieve optimal entanglement generation and we show that they are reachable with current experimental technology.

PACS numbers: 03.67.Bg, 03.65.Yz, 42.50.Dv, 42.50.Pq
\end{abstract}

\section{INTRODUCTION}

The Dicke model describes the dynamics of $N$ identical two-level atoms interacting with a quantized threedimensional electromagnetic (EM) field [1]. Under certain conditions the model predicts that the atoms interact with the quantized EM field collectively, giving rise to the widely studied phenomena of superradiance and subradiance 2, 3]. In free space ideal superradiance and subradiance take place in the so called small sample limit, i.e., when the atoms are so close to each other that one can ignore any effect resulting from their different spatial positions. In this case the atoms are indistinguishable with respect to their emission and absorption properties; hence, the presence of equivalent paths through which the emission process may occur gives rise to fully constructive (superradiance) or destructive (subradiance) interference.

Ideal superradiance or subradiance in free space is very difficult to observe in the experiments since it requires that the atoms are placed in a regular pattern within a sample smaller than the wavelength of the EM field they interact with (small sample case). The requirement of a regular pattern is due to the presence of the dipoledipole forces that would otherwise break the symmetry under permutation of any two atoms necessary to observe superradiant-subradiant behavior. Such a regularity can be achieved, e.g., with trapped-ion crystals [4] or atoms in optical lattices [5]. In these systems, however, the separation between the particles is typically larger or on the same order of magnitude than the resonant wavelength (large sample case). In the large sample case, cooperative effects still occur but the subradiant state is not completely decoupled from the dynamics. Indeed, partial subradiance and superradiance have been observed with trapped ions [6].

A way for relaxing the requirement for configuration regularity is to place the small sample in a cavity resonator. In this case, indeed, due to the Purcell effect,

*Electronic address: kari.harkonen@utu.fi the cooperative atomic behavior can be observed at much lower atomic density than in free space, making the van der Waals dephasing caused by the irregular atomic configuration negligible 2]. Experiments observing superradiance in the small sample case in a cavity have been performed with Rydberg atoms [7], giving results in a very good agreement with the predictions of the singlemode superradiance theory. In this experiment, all of the atoms are equivalently coupled to the quantized mode of the EM field (homogeneous case).

Recent advances in ion-cavity QED experiments make it possible to confine arrays of ions inside an optical cavity in a regime in which the width of their wave packet in position space is smaller than the wavelength of the cavity mode they interact with (Lamb-Dicke regime) [8, 9]. Moreover, it is possible to accurately manipulate the position of the single ions with respect to the intensity profile of the standing cavity mode, thereby allowing us to change the strength of the coupling between each ion and the quantized EM field.

It has been demonstrated theoretically that, when the atoms are coupled with different strengths to the EM field, ideal superradiance or subradiance can still occur, depending on the particular spatial distribution of the atoms [10, 11, 12]. However, no experiments have up to now confirmed these predictions by the inhomogeneous Dicke model. Very recently, an important step in this direction has been achieved at the University of Aarhus, where a collective strong coupling between an ion crystal and a cavity mode was observed [13]. In this paper, we investigate in detail how the inhomogeneous singlemode Dicke model (or Tavis-Cummings model [14]) can be realized in the ion-cavity QED context and the conditions under which subradiance and superradiance can be observed.

Besides the importance in the study of fundamentals of quantum theory, the realization of the Dicke model and the generation of the subradiant state play a crucial role in quantum information technology and quantum communication. Indeed, arrays of ions are ideal candidates for quantum registers and their controlled interaction with photons allows us to realize atom-light quantum interfaces [15] and to distribute entanglement to different 
nodes of quantum networks. The importance of the subradiant states in this context stems from the fact that they are robust entangled atomic states since they are completely decoupled from the EM field.

The aim of this work is to discuss a realistic setup that is able to show the collective behavior of trapped ions in a cavity. In particular, since in the experiments performed so far the ions are coupled to the EM mode via a Raman scheme in a $\Lambda$-configuration, we will include the entire level structure, which is important in order to understand the decohering role of the spontaneous emission from the upper and essentially unpopulated level. We will also include cavity losses in order to study in detail the deviation from the ideal cooperative Dicke model and to identify the parameter regions in which such deviations are as small as possible.

In fact, during the last two decades, several theoretical papers have discussed issues such as entanglement generation, preparation of nonclassical states, or realization of quantum gates in the ion-cavity QED context assuming that the conditions to realize an ideal Tavis-Cummings model were met [16, 17, 18, 19, 20, 21, 22, 23, 24, 25, 26]. Thus, either the spontaneous emission or the cavity losses (or both processes) are usually neglected [17, 19, 23, 24. Concerning spontaneous emission, for example, the assumption is made that the emission rate is much smaller than the cavity coupling constant [16, 20, 21, 22, 25]. However, this condition is not met in the ion-cavity QED experiments [8, 9]. Furthermore, as we will demonstrate in this paper, if one deals with simplified atomic level structures [18, 19, 26, 27], it is not possible to single out those regions in parameter space for which the systems of trapped ions behave collectively.

In this paper, we will take both the cavity losses and the spontaneous emissions into account and employ $\Lambda$ type schemes to describe the ions and to identify the experimental conditions under which the coherent dynamics predicted by the single-mode Dicke model is dominant with respect to losses and decoherence. This will also allow us to present realistic protocols for entanglement generation and to discuss ways to optimize the generated entanglement using specific features of the trapped-ion system, such as the ability to manipulate in a controlled way the relative coupling between the ions and the cavity field.

The structure of the paper is the following. In Sec. II we review the properties of the inhomogeneous singlemode Dicke model. In Sec. III we present the Hamiltonian for two ions in a cavity and we make the connection to the Dicke model by deriving an effective model describing the dynamics under realistic experimental conditions. Section IV is devoted to the description of the experimental proposal to observe subradiance and verify the inhomogeneous Dicke model. Furthermore, in Sec.V we explore another way to optimize the entanglement generation by using off-resonant transitions. Finally, a summary of the results and the conclusions are given in Sec. VI.

\section{INHOMOGENEOUS SINGLE-MODE DICKE MODEL}

\section{A. Ideal cavity}

The single-mode Dicke model, or Tavis-Cummings model, is the simplest quantum-mechanical model describing collective effects such as superradiance and subradiance in cavity. It describes the quasi-resonant interaction between $N$ identical two-level atoms and a single quantized cavity mode. The Tavis-Cummings Hamiltonian is

$$
\begin{aligned}
H_{\mathrm{D}}= & \omega_{C}\left(a^{\dagger} a+\frac{1}{2}\right)+\sum_{j=1}^{N} \omega_{A} \sigma_{+}^{(j)} \sigma_{-}^{(j)} \\
& +\sum_{j=1}^{N}\left(\alpha^{(j)} a^{\dagger} \sigma_{-}^{(j)}+\alpha^{(j) *} a \sigma_{+}^{(j)}\right),
\end{aligned}
$$

where $\omega_{C}$ and $\omega_{A}$ are the frequencies of the cavity mode and the atomic transition, respectively; $a$ and $a^{\dagger}$ are the annihilation and the creation operators for the cavity mode; and $\sigma_{-}^{(j)}=\left|0^{(j)}\right\rangle\left\langle 1^{(j)}\right|$ and $\sigma_{+}^{(j)}=\left(\sigma_{-}^{(j)}\right)^{\dagger}$ are the lowering and the raising operators for the $j$ th atom, $\left|0^{(j)}\right\rangle$ and $\left|1^{(j)}\right\rangle$ being its ground and excited states, respectively. Finally, $\alpha^{(j)}$ is the coupling strength of the $j$ th atom with the cavity field. Inhomogeneity of the coupling strengths originates from different relative positions of the atoms with respect to the intensity profile of the standing EM mode supported by the cavity resonator.

This model assumes that the cavity is ideal, as photon escape is not taken into account, and that atomic spontaneous emission from the excited to the ground state is negligible. The model also neglects the atomic motion as well as recoil effects due to the absorption and subsequent re-emission of a photon by the atoms. Moreover, the dipolar coupling of the atoms and the EM field is expressed within a rotating wave approximation (RWA), thereby suppressing the non-energy-conserving terms. Finally, it implicitly assumes that the coupling between the atoms and the cavity mode does not change, i.e., that the atoms are kept at fixed positions. While the RWA has been proven to work extremely well in optical experiments, all other assumptions need further consideration. In the following sections we will examine them in detail for the ion-cavity QED setup.

Using a suitable canonical transformation it has been shown that, when only one excitation is present in the total system, the $N$ atoms interacting with the quantized field mode according to Eq. (1) cooperate in such a way that only one collective atomic mode (superradiant state) is coupled to the field [11]. Consequently, the energy exchange between the atoms and the field can be completely suppressed if the only field-coupled collective mode is unexcited.

For simplicity, we will from now on focus on the $N=2$ case sketched in Fig. 1] and we will denote the energy eigenstates for the free ions as $\left|a^{(1)} b^{(2)}\right\rangle \equiv\left|a^{(1)}\right\rangle \otimes\left|b^{(2)}\right\rangle$ 


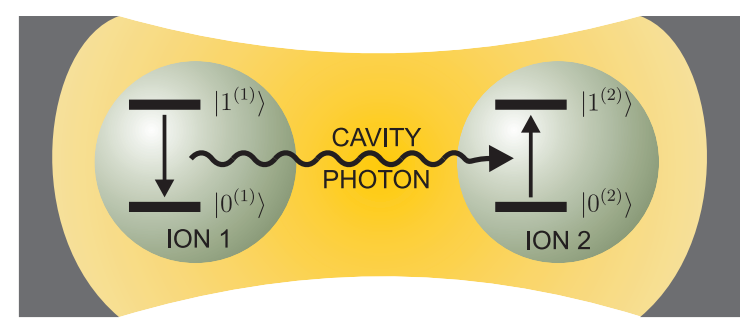

FIG. 1: (Color online) Two binary quantum objects interacting through a quantized electromagnetic mode supported by a cavity resonator. The dynamics of such an ideal system is described by the Dicke model.

(with $a, b=0,1$ ) and the corresponding Fock states of the cavity mode as $\left|n^{(C)}\right\rangle$, where $n=0,1, \ldots$. The time evolution generated by $H_{D}$ is easily obtained explicitly. For a cavity initially prepared in the vacuum state, and in the presence of only one atomic excitation, the time evolution of the amplitudes $c_{10}(t)$ and $c_{01}(t)$ to find the ions in the states $\left|1^{(1)} 0^{(2)}\right\rangle$ and $\left|0^{(1)} 1^{(2)}\right\rangle$, respectively, is given by

$$
\begin{aligned}
c_{10}(t)= & {\left[\left|r^{(2)}\right|^{2}+\left|r^{(1)}\right|^{2} \mathcal{E}(t)\right] c_{10}(0) } \\
& -r^{(1) *} r^{(2)}[1-\mathcal{E}(t)] c_{01}(0), \\
c_{01}(t)= & -r^{(1)} r^{(2) *}[1-\mathcal{E}(t)] c_{10}(0) \\
& +\left[\left|r^{(1)}\right|^{2}+\left|r^{(2)}\right|^{2} \mathcal{E}(t)\right] c_{01}(0) .
\end{aligned}
$$

In the equations above the relative coupling strengths are defined as $r^{(j)}=\alpha^{(j)} /\left|\alpha_{T}\right|$, where $\left|\alpha_{T}\right|=$ $\sqrt{\left|\alpha^{(1)}\right|^{2}+\left|\alpha^{(2)}\right|^{2}}$ is the total coupling strength, and

$$
\mathcal{E}(t)=e^{i \delta t / 2}\left[\cos \left(\frac{\Omega_{\mathrm{v}} t}{2}\right)-i \frac{\delta}{\Omega_{\mathrm{v}}} \sin \left(\frac{\Omega_{\mathrm{v}} t}{2}\right)\right],
$$

where $\delta=\omega_{A}-\omega_{C}$ is the detuning and $\Omega_{\mathrm{v}}=$ $\sqrt{4\left|\alpha_{T}\right|^{2}+\delta^{2}}$ is the vacuum Rabi frequency. Note that $r^{(1)}$ and $r^{(2)}$ are not independent parameters, since $\left|r^{(2)}\right|=\sqrt{1-\left|r^{(1)}\right|^{2}}$.

The subradiant $\left|\psi_{-}\right\rangle$and the superradiant $\left|\psi_{+}\right\rangle$states are

$$
\begin{aligned}
& \left|\psi_{-}\right\rangle=r^{(2)}\left|1^{(1)} 0^{(2)}\right\rangle-r^{(1)}\left|0^{(1)} 1^{(2)}\right\rangle, \\
& \left|\psi_{+}\right\rangle=r^{(1) *}\left|1^{(1)} 0^{(2)}\right\rangle+r^{(2) *}\left|0^{(1)} 1^{(2)}\right\rangle,
\end{aligned}
$$

and, in this case, they are position dependent through the relative coupling strength parameters $r^{(1)}$ and $r^{(2)}$. As one can see directly from Eq. (11), the state $\left|\psi_{-}\right\rangle \otimes\left|0^{(C)}\right\rangle$ is an eigenstate of the Tavis-Cummings Hamiltonian with eigenvalue $\frac{1}{2} \omega_{C}+\omega_{A}$. Therefore, when the atoms are prepared in this state, they are completely decoupled from the cavity field and the system does not evolve at all. In the case of equally strong couplings, i.e., for $\left|r^{(1)}\right|=\left|r^{(2)}\right|=1 / \sqrt{2}$, the subradiant and the superradiant states coincide with the maximally entangled Bell states. In general, however, these states are not maximally entangled.

\section{B. Non-ideal cavity}

We now proceed to generalize Eq. (1) to the case of a lossy cavity. The imperfect reflectivity of the cavity mirrors and consequent leakage of photons causes a Lorentzian broadening of the spectral line corresponding to the mode supported by the ideal cavity. Accordingly, the microscopic atom-field interaction should now take into account a continuum of modes described by a Lorentzian distribution peaked at the central cavity frequency $\omega_{C}$. For the sake of simplicity, and in view of the discussion in the ion-cavity QED context, we restrict our attention to a one-dimensional cavity model. Namely, we neglect the coupling with all the EM modes other than the ones supported by the lossy cavity. In the rotating wave approximation, the Hamiltonian is given by

$$
\begin{aligned}
H= & \sum_{k} \omega_{k}\left(a_{k}^{\dagger} a_{k}+\frac{1}{2}\right)+\sum_{j=1}^{N} \omega_{A} \sigma_{+}^{(j)} \sigma_{-}^{(j)} \\
& +\sum_{k} \sum_{j=1}^{N}\left[i g_{k} \sin \left(\frac{\omega_{k}}{c} x^{(j)}\right) a_{k}^{\dagger} \sigma_{-}^{(j)}+H . c .\right]
\end{aligned}
$$

where $a_{k}$ and $a_{k}^{\dagger}$ are the annihilation and the creation operators of cavity photons of frequency $\omega_{k}$, respectively. Above, we have assumed that all the atoms have the same electric dipole moment, which has been incorporated in the coupling constants $g_{k}$, and we indicate with $x^{(j)}$ the position of the atoms along the cavity axis. In the following we will assume that each atom is kept at a fixed position inside the cavity and that they are all well localized, i.e., the spread of their wave function in position space is smaller than the wavelength of the central cavity field mode: $\Delta x^{(j)} \ll c / \omega_{C}$. Since all the significantly contributing modes are close to the central mode (of frequency $\omega_{C}$ ), we have

$$
\sin \left(\frac{\omega_{k}}{c} x^{(j)}\right) \simeq \sin \left(\frac{\omega_{C}}{c} x^{(j)}\right),
$$

and Eq. (7) takes the form

$$
\begin{aligned}
H= & \sum_{k} \omega_{k}\left(a_{k}^{\dagger} a_{k}+\frac{1}{2}\right)+\sum_{j=1}^{N} \omega_{A} \sigma_{+}^{(j)} \sigma_{-}^{(j)} \\
& +\sum_{j=1}^{N}\left[\chi^{(j)} \sigma_{-}^{(j)} \sum_{k} g_{k} a_{k}^{\dagger}+H . c .\right]
\end{aligned}
$$

with $\chi^{(j)}=i \sin \left(\omega_{C} x^{(j)} / c\right)$. In the continuum limit the sum over the $k$-modes is replaced with an integral

$$
\sum_{k}\left|g_{k}\right|^{2} \rightarrow \int d \omega J(\omega)
$$

where $J(\omega)$ is the reservoir spectral density. As mentioned above, we assume a Lorentzian distribution for 
the spectrum of the field inside the cavity; therefore, we take a spectral density of the form

$$
J(\omega)=\frac{W^{2}}{2 \pi} \frac{\kappa}{\left(\omega-\omega_{C}\right)^{2}+(\kappa / 2)^{2}},
$$

where the distribution is characterized by its full width at half maximum value $\kappa$ and by a normalization parameter $W^{2}=\int d \omega J(\omega)$. Hence, $\kappa$ describes the cavity losses and $W$ describes the total coupling strength.

We focus again on the two-atom case, i.e., $N=2$, and we consider the situation in which only one excitation is present in the total atoms-field system. Starting from the Hamiltonian (77) and using the Lorentzian spectral density (10), it is possible to derive an effective master equation

$$
\frac{d \varrho}{d t}=-i\left[H_{D}, \varrho\right]-\frac{\kappa}{2}\left[a^{\dagger} a \varrho+\varrho a^{\dagger} a-2 a \varrho a^{\dagger}\right]
$$

for the dynamics of the atoms and the cavity mode of frequency $\omega_{C}$ [28]. Here, $a$ and $a^{\dagger}$ are the annihilation and the creation operators for the central cavity mode, which is damped at rate $\kappa$, and the coherent dynamics is generated by $H_{D}$ in Eq. (1), where the coupling constants are identified as $\alpha^{(j)}=\chi^{(j)} W$. From the exact solution of the effective master equation (11), one can obtain the state of the atomic system by tracing out the cavity degree of freedom: $\rho(t)=\operatorname{tr}_{C}[\varrho(t)]$.

After performing the trace, and for an initially empty cavity, the problem can be solved exactly. In the ordered basis $\left\{\left|1^{(1)} 1^{(2)}\right\rangle,\left|1^{(1)} 0^{(2)}\right\rangle,\left|0^{(1)} 1^{(2)}\right\rangle,\left|0^{(1)} 0^{(2)}\right\rangle\right\}$, the atomic density matrix can be written in the form [29]

$$
\rho(t)=\left(\begin{array}{cccc}
0 & 0 & 0 & 0 \\
0 & \left|c_{10}\right|^{2} & c_{10} c_{01}^{*} & 0 \\
0 & c_{10}^{*} c_{01} & \left|c_{01}\right|^{2} & 0 \\
0 & 0 & 0 & 1-\left|c_{10}\right|^{2}-\left|c_{01}\right|^{2}
\end{array}\right) .
$$

The dynamics of the two qubits is therefore completely characterized by the two amplitudes:

$$
\begin{aligned}
c_{10}(t)= & {\left[\left|r^{(2)}\right|^{2}+\left|r^{(1)}\right|^{2} \mathcal{E}(t)\right] c_{10}(0) } \\
& -r^{(1) *} r^{(2)}[1-\mathcal{E}(t)] c_{01}(0), \\
c_{01}(t)= & -r^{(1)} r^{(2) *}[1-\mathcal{E}(t)] c_{10}(0) \\
& +\left[\left|r^{(1)}\right|^{2}+\left|r^{(2)}\right|^{2} \mathcal{E}(t)\right] c_{01}(0),
\end{aligned}
$$

with $r^{(j)}=\chi^{(j)} /\left|\chi_{T}\right|$, where $\left|\chi_{T}\right|=\sqrt{\left|\chi^{(1)}\right|^{2}+\left|\chi^{(2)}\right|^{2}}$, and

$\mathcal{E}(t)=e^{-(\kappa-i 2 \delta) t / 4}\left[\cos \left(\frac{\Omega_{g} t}{2}\right)+\frac{\kappa-i 2 \delta}{2 \Omega_{g}} \sin \left(\frac{\Omega_{g} t}{2}\right)\right]$,

where $\Omega_{g}=\sqrt{4\left|\chi_{T}\right|^{2} W^{2}+\delta^{2}+i \delta \kappa-\kappa^{2} / 4}$ is the generalized Rabi frequency. Note that Eqs. (13) and (14) have exactly the same structure as Eqs. (2) and (3), obtained for the single-mode Dicke model without losses. Formally, the cavity losses appear as an additional imaginary part of the detuning $\delta \mapsto \delta+i \kappa / 2$. Accordingly, the effect of the cavity losses is described by the modification of the time-dependent coefficient $\mathcal{E}(t)$, which is now damped at rate $\kappa / 4$, and by the $\kappa$-dependent shift of the Rabi frequency. For $\kappa \rightarrow 0$, the Lorentzian spectral density (10) tends to Dirac's delta distribution, $J(\omega) \rightarrow W^{2} \delta\left(\omega-\omega_{C}\right)$, and Eq. (15) reduces to Eq. (4), with $\alpha^{(j)}=\chi^{(j)} W$.

It is worth noticing that, as one sees directly from Eq. (9), the subradiant state $\left|\psi_{-}\right\rangle$, given by Eq. (5), is still decoupled from the vacuum cavity field. Hence, if the atomic system is initially prepared in this state, no exchange of excitation with the cavity field will take place.

\section{EFFECTIVE MODEL OF ION-CAVITY INTERACTION}

\section{A. Physical setup}

Ion-cavity QED experiments use calcium ions which are trapped in a linear Paul microtrap and interact with a quantized mode of a high-finesse optical cavity [8, 9]. In Fig. 2 we show the relevant energy-level structure, couplings, and decay channels for the compound system of two ${ }^{40} \mathrm{Ca}^{+}$ions and a single cavity mode. The atomic ground state $4^{2} S_{1 / 2}$ is coupled to the electronically excited state $4^{2} P_{1 / 2}$ by a (classical) pump laser injected from the side of the cavity. On the other hand, the excited state $4^{2} P_{1 / 2}$ is coupled to a metastable state $3^{2} D_{3 / 2}$ by the quantized cavity mode. The excited state $4^{2} P_{1 / 2}$ decays spontaneously to the states $4^{2} S_{1 / 2}$ and $3^{2} D_{3 / 2}$ at rates $\gamma_{S}$ and $\gamma_{D}$, respectively, and the cavity photon is damped at rate $\kappa$, as explained in the previous section.

A realistic theoretical description of the dynamics of a single ${ }^{40} \mathrm{Ca}^{+}$ion coupled to the cavity mode has been given in Ref. 30]. The authors consider there also the effect of cavity losses and spontaneous emission, taking into account all the Zeeman sublevels of the three relevant electronic states. The main consequence of the presence of the Zeeman sublevels is a reduction in the coupling driven by the cavity field by a factor of $\sqrt{3}$ with respect to the simpler three-level model considered here. Therefore, we will use in the following a three-level model scheme with such a reduced effective coupling to account for the presence of the Zeeman sublevels. In the experiments, the ions sit at the bottom of the trapping potential and are cooled down to the Lamb-Dicke regime. Under these conditions one can assume that the ions are kept at fixed positions and neglect recoil during the emissionabsorption process.

In the following we will consider as initial atomic states those in which one of the two atoms is in its ground state and the other one is in its metastable state, i.e., the states $\left|S^{(1)} D^{(2)}\right\rangle$ and $\left|D^{(1)} S^{(2)}\right\rangle$. In order to prepare these states, if the vibrational sidebands are not resolved, 

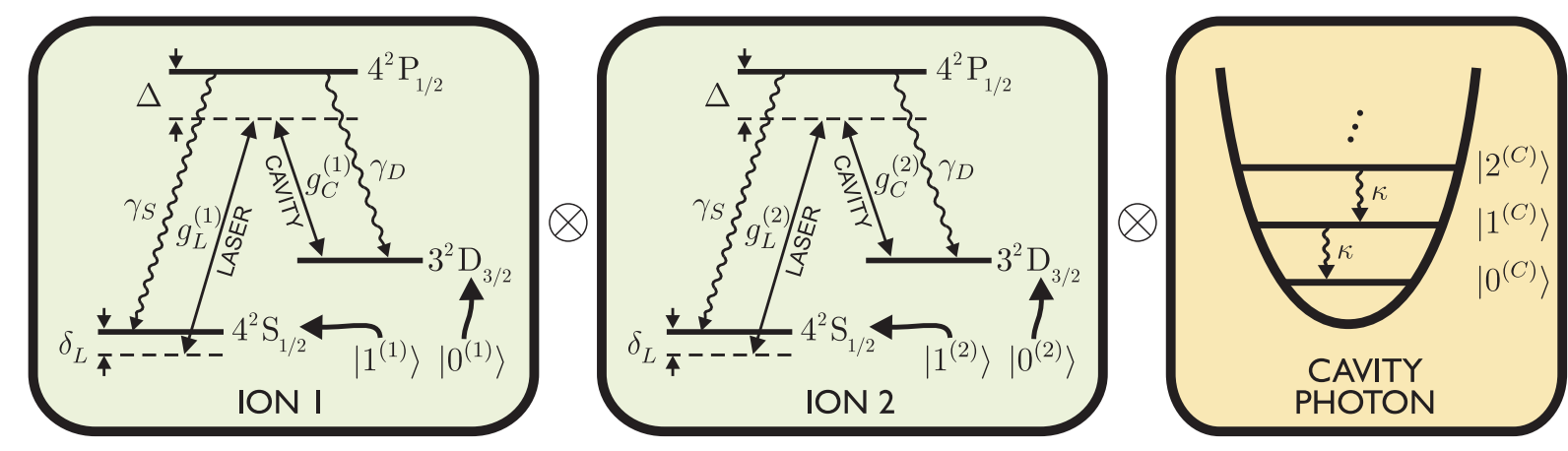

FIG. 2: (Color online) The relevant electronic states of two identical ${ }^{40} \mathrm{Ca}^{+}$ions and corresponding couplings provided by an external pump laser and a quantized cavity mode. The excited electronic state decays spontaneously to the ground and to the metastable states, and the cavity mode is damped as well.

it is necessary to use a selective laser addressing of the individual ions. This is routinely done in trapped-ion experiments with ${ }^{40} \mathrm{Ca}^{+}$ions (see, e.g., 31] ).

The two identical ions interact with the quantized cavity mode of frequency $\omega_{C}$ via laser-assisted two-photon processes, as shown in Fig. 2 The ions are irradiated by a laser beam of frequency $\omega_{L}+\delta_{L}$. The laser beams and the cavity field are far detuned by $\Delta$ from the electronic level $\left|P^{(j)}\right\rangle$, such that $\omega_{P}-\omega_{D}=\omega_{C}+\Delta$ and $\omega_{P}-\omega_{S}=\omega_{L}+\Delta$. Therefore, the setup provides each ion $j=1,2$ with a Raman coupling between the levels $\left|S^{(j)}\right\rangle$ and $\left|D^{(j)}\right\rangle$.

The time evolution of the composite system of the two ions and the cavity mode can be described by a master equation

$$
\begin{aligned}
\frac{d \varrho}{d t}= & -i[H(t), \varrho]-\frac{\kappa}{2}\left(a^{\dagger} a \varrho+\varrho a^{\dagger} a-2 a \varrho a^{\dagger}\right) \\
& -\frac{\gamma_{S}}{2} \sum_{j=1,2}\left(A_{P P}^{(j)} \varrho+\varrho A_{P P}^{(j)}-2 A_{S P}^{(j)} \varrho A_{P S}^{(j)}\right) \\
& -\frac{\gamma_{D}}{2} \sum_{j=1,2}\left(A_{P P}^{(j)} \varrho+\varrho A_{P P}^{(j)}-2 A_{D P}^{(j)} \varrho A_{P D}^{(j)}\right)
\end{aligned}
$$

where we have included the cavity field damping at rate $\kappa$, the spontaneous emission channels (two for each ion) at rates $\gamma_{S}$ and $\gamma_{D}$, and where the coherent dynamics is generated by a Hamiltonian

$$
\begin{aligned}
& H(t)=\omega_{C}\left(a^{\dagger} a+\frac{1}{2}\right)+\sum_{j=1,2} \sum_{l=S, P, D} \omega_{l} A_{l l}^{(j)} \\
& +\sum_{j=1,2}\left(e^{-i\left(\omega_{L}+\delta_{L}\right) t} g_{L}^{(j)} A_{P S}^{(j)}+g_{C}^{(j)} a A_{P D}^{(j)}+H . c .\right) .
\end{aligned}
$$

The atomic operators are defined as $A_{l l^{\prime}}^{(j)}=\left|l^{(j)}\right\rangle\left\langle l^{\prime(j)}\right|$, with $l, l^{\prime}=S, P, D$ and $j=1,2$. Finally, the coherent couplings provided by the laser and the cavity mode are, respectively,

$$
\begin{aligned}
& g_{L}^{(j)}=\Omega e^{i k_{L} x^{(j)}}, \\
& g_{C}^{(j)}=g \sin \left(k_{C} x^{(j)}\right),
\end{aligned}
$$

with $k_{L}$ and $k_{C}$ being the wave numbers of the laser and the standing cavity mode.

\section{B. Effective two-level model}

When the detuning $\Delta$ is sufficiently large compared to the couplings, $\Delta \gg g_{L}^{(j)}, g_{C}^{(j)}$, the excited electronic states $\left|P^{(j)}\right\rangle$ can be adiabatically eliminated from the dynamics, as described in detail in Appendix A. In this case the system can be effectively described as composed of two two-level atoms interacting with a cavity mode. For this purpose, we denote the ground and the metastable states of the $j$ th atom as $\left|1^{(j)}\right\rangle \equiv\left|S^{(j)}\right\rangle$ and $\left|0^{(j)}\right\rangle \equiv\left|D^{(j)}\right\rangle$ (N.B., the true atomic ground state corresponds to the excited state of the effective two-level system, since it is able to emit a cavity photon through the Raman transition).

The adiabatic elimination of the excited levels $\left\{\left|P^{(j)}\right\rangle\right\}$ is not at all trivial due to the inclusion of the spontaneous emission processes [32. We show in Appendix A that an effective Tavis-Cummings Hamiltonian can be derived, describing an excitation exchange between the ions and the cavity. However, one needs to include (i) two Stark shift terms per ion (one, in particular, being dependent on the state of the cavity mode) and (ii) an overall rescaling of both the free and the interaction energies by a factor explicitly dependent on the emission rates.

It turns out that, in the interaction picture with respect to $H_{0}-\Delta \sum_{j} A_{P P}^{(j)}$, where $H_{0}$ is given by the first two terms on the right-hand side of Eq. (17), the coherent part of the evolution of the ion-cavity system is described 
by an effective Hamiltonian

$$
\begin{aligned}
H_{\mathrm{eff}}= & -\xi \sum_{j=1,2}\left[\left(e^{-i \delta_{L} t} \frac{\beta^{(j)} g^{*} \Omega}{\Delta} a^{\dagger} A_{01}^{(j)}+H . c .\right)\right. \\
& \left.+\frac{\left|\beta^{(j)} g\right|^{2}}{\Delta} a^{\dagger} a A_{00}^{(j)}+\frac{|\Omega|^{2}}{\Delta} A_{11}^{(j)}\right]
\end{aligned}
$$

where the position-dependent parameters $\beta^{(j)}$ are defined as

$$
\beta^{(j)}=e^{i k_{L} x^{(j)}} \sin \left(k_{C} x^{(j)}\right),
$$

and the dimensionless renormalizing prefactor is

$$
\xi=\frac{\Delta^{2}}{\Delta^{2}+\left(\gamma_{S}+\gamma_{D}\right)^{2} / 4} .
$$

This Hamiltonian resembles the Tavis-Cummings Hamiltonian (1), except for the photon-dependent Stark shift term. However, since the original microscopic model includes dissipative processes, the unitary evolution generated by $H_{\text {eff }}$ needs to be supplemented by decohering terms that have a very peculiar structure. Indeed, the effective master equation that describes the time evolution of the ions and the cavity contains four (now both dissipative and non-dissipative) processes (described by jump operators) that take into account the effects of the spontaneous emission as seen in the restricted atomic subspaces spanned by $\left\{\left|0^{(j)}\right\rangle,\left|1^{(j)}\right\rangle\right\}$. The cavity damping appears in the restricted subspace in the same form as in the original model.

The effective master equation reads

$$
\begin{gathered}
\frac{d \varrho}{d t}=-i\left[H_{\mathrm{eff}}, \varrho\right]-\frac{\kappa}{2}\left(a^{\dagger} a \varrho+\varrho a^{\dagger} a-2 a \varrho a^{\dagger}\right) \\
-\sum_{\substack{j=1,2 \\
m=S, D}} \frac{\Gamma_{m}^{(j)}}{2}\left[C_{m}^{(j) \dagger} C_{m}^{(j)} \varrho+\varrho C_{m}^{(j) \dagger} C_{m}^{(j)}\right. \\
\left.-2 C_{m}^{(j)} \varrho C_{m}^{(j) \dagger}\right]
\end{gathered}
$$

where the jump operators for each ion $j$ are given by

$$
\begin{aligned}
& C_{S}^{(j)}=e^{-i \delta_{L} t} \Omega A_{11}^{(j)}+\beta^{(j) *} g a A_{10}^{(j)}, \\
& C_{D}^{(j)}=e^{-i \delta_{L} t} \Omega A_{01}^{(j)}+\beta^{(j) *} g a A_{00}^{(j)},
\end{aligned}
$$

while the effective decay rates are $\Gamma_{m}^{(j)}=\xi \gamma_{m} / \Delta^{2}$, where $m=S, D$ and the prefactor $\xi$ is given by Eq. (22). The structure of these jump operators is easy to interpret once the full level configurations of Fig. 2 are taken into account. Let us consider, for example, the operator $C_{S}^{(j)}$ of Eq. (24). It arises from the spontaneous emission process $4^{2} P_{1 / 2} \rightarrow 4^{2} S_{1 / 2}$ of the $j$ th atom, now being restricted to the two-dimensional subspace $\left\{\left|0^{(j)}\right\rangle,\left|1^{(j)}\right\rangle\right\}$. The jump operator $C_{S}^{(j)}$ has two contributions, both of them describing non-dissipative decoherence by pure dephasing processes (as one understands from the fact that they do not produce any excitation loss). These two contributions account for the interruption of the ion-cavity excitation exchange (vacuum Rabi cycle) by the spontaneous emission. The first term is an unwanted repopulation of state $\left|1^{(j)}\right\rangle$ occurring after the laser has virtually brought the system to the intermediate level $\left|P^{(j)}\right\rangle$ of the full Raman cycle. The second term is also due to decay into state $\left|1^{(j)}\right\rangle$, but this time the virtual excitation of level $\left|P^{(j)}\right\rangle$ is caused by the cavity field. In conclusion, both processes interrupt the vacuum Rabi cycle without the excitation being lost as, at the end, the two-level system is found in its excited state $\left|1^{(j)}\right\rangle$. This implies that the excitation exchange can restart, but with a different phase. Thus, $C_{S}^{(j)}$ describes a phase error.

A similar interpretation scheme can be adopted for the two terms constituting $C_{D}^{(j)}$ in Eq. (25). However, this time the involved process is the spontaneous emission $4^{2} P_{1 / 2} \rightarrow 3^{2} D_{3 / 2}$. Whether it occurs after the virtual excitation of level $\left|P^{(j)}\right\rangle$ performed by the laser (first term) or by the cavity field (second term), the result is that at the end the two-level system is found in its ground state $\left|0^{(j)}\right\rangle$ and that one excitation has been lost either from the atom or from the cavity mode. Therefore, this jump operator causes dissipative decoherence. We note that, at this stage, the four jump operators of Eqs. (24)-(25) are both explicitly time dependent and implicitly position dependent via the coefficients $\beta^{(j)}$ [see Eq. (21)].

A phase rotation within the restricted Hilbert space, spanned by the states with at maximum one excitation, allows transforming the effective Hamiltonian (20) into the Tavis-Cummings Hamiltonian (1) as well as removing simultaneously the time dependence from the jump operators (24) and (25). This is described in Appendix B. Therefore, in a suitable rotating frame, the following effective Tavis-Cummings Hamiltonian is obtained:

$$
\begin{aligned}
H_{D}^{\mathrm{eff}}= & \omega_{C}^{\mathrm{eff}}\left(a^{\dagger} a+\frac{1}{2}\right)+\sum_{j=1,2} \omega_{A}^{\mathrm{eff}} \sigma_{+}^{(j)} \sigma_{-}^{(j)} \\
& +\sum_{j=1,2}\left(\alpha_{\mathrm{eff}}^{(j)} a^{\dagger} \sigma_{-}^{(j)}+\alpha_{\mathrm{eff}}^{(j) *} a \sigma_{+}^{(j)}\right),
\end{aligned}
$$

where we have introduced again the spin inversion operators used in Sec. III The effective Dicke model parameters are

$$
\begin{aligned}
& \omega_{C}^{\mathrm{eff}}=-\xi \frac{2\left|\beta_{T} g\right|^{2}}{3 \Delta}, \\
& \omega_{A}^{\mathrm{eff}}=\delta_{L}-\xi\left(\frac{|\Omega|^{2}}{\Delta}-\frac{\left|\beta_{T} g\right|^{2}}{3 \Delta}\right), \\
& \alpha_{\mathrm{eff}}^{(j)}=-\xi \frac{\beta^{(j)} g^{*} \Omega}{\Delta} \equiv \beta^{(j)} g_{\mathrm{eff}},
\end{aligned}
$$

where $\left|\beta_{T}\right|=\sqrt{\left|\beta^{(1)}\right|^{2}+\left|\beta^{(2)}\right|^{2}}$. The effective detuning is given by

$$
\delta_{\mathrm{eff}}=\omega_{A}^{\mathrm{eff}}-\omega_{C}^{\mathrm{eff}}=\delta_{L}-\xi \frac{|\Omega|^{2}-\left|\beta_{T} g\right|^{2}}{\Delta},
$$


and the relative effective coupling strengths $r^{(j)}$ [cf. Eqs. (2) and(3)] are directly given by the position-dependent parameters $\beta^{(j)}$, since now $r^{(j)}=$ $\alpha_{\text {eff }}^{(j)} /\left|\alpha_{\text {eff, } T}\right|=\beta^{(j)} /\left|\beta_{T}\right|$.

Comparing Eqs. (26) and (23) with Eqs. (1) and (11), respectively, we see that, when the effective atomic spontaneous emissions are negligible, this system allows us to realize the Dicke model in the non-ideal cavity case.

\section{Effective spontaneous emission processes}

As mentioned before, we restrict our study to the case in which only one or zero quanta are present in the composite system of the two ions and the cavity mode. Therefore, the compound state of the two atoms and the cavity photon can be expressed in the basis $\left\{\left|0^{(1)} 0^{(2)} 0^{(C)}\right\rangle\right.$, $\left.\left|0^{(1)} 0^{(2)} 1^{(C)}\right\rangle, \quad\left|0^{(1)} 1^{(2)} 0^{(C)}\right\rangle, \quad\left|1^{(1)} 0^{(2)} 0^{(C)}\right\rangle\right\} \quad$ (see Appendix B). Consequently, the jump operators (24)-(25) can be normalized with respect to the operator norm $\|A\|=\sup _{\|\phi\|=1} \| A|\phi\rangle \|$, where $|\phi\rangle$ belongs to the Hilbert space spanned by the basis defined above. The introduction of the normalized jump operators allows to define the effective spontaneous emission decay rates $\Gamma_{m}^{(j)}$ unambiguously.

The normalized jump operators are

$$
\begin{aligned}
& C_{S}^{(1)}=\left|1^{(1)} 0^{(2)} 0^{(C)}\right\rangle\left\langle\Phi_{1}\right|, \\
& C_{S}^{(2)}=\left|0^{(1)} 1^{(2)} 0^{(C)}\right\rangle\left\langle\Phi_{2}\right|, \\
& C_{D}^{(1)}=\left|0^{(1)} 0^{(2)} 0^{(C)}\right\rangle\left\langle\Phi_{1}\right|, \\
& C_{D}^{(2)}=\left|0^{(1)} 0^{(2)} 0^{(C)}\right\rangle\left\langle\Phi_{2}\right|,
\end{aligned}
$$

where the decaying states are

$$
\begin{aligned}
\left|\Phi_{1}\right\rangle & =\frac{\Omega^{*}\left|1^{(1)} 0^{(2)} 0^{(C)}\right\rangle+\beta^{(1)} g^{*}\left|0^{(1)} 0^{(2)} 1^{(C)}\right\rangle}{\sqrt{|\Omega|^{2}+\left|\beta^{(1)} g\right|^{2}}}, \\
\left|\Phi_{2}\right\rangle & =\frac{\Omega^{*}\left|0^{(1)} 1^{(2)} 0^{(C)}\right\rangle+\beta^{(2)} g^{*}\left|0^{(1)} 0^{(2)} 1^{(C)}\right\rangle}{\sqrt{|\Omega|^{2}+\left|\beta^{(2)} g\right|^{2}}} .
\end{aligned}
$$

The corresponding rescaled decay rates are given by

$$
\begin{aligned}
& \Gamma_{S}^{(j)}=\xi\left(|\Omega|^{2}+\left|\beta^{(j)} g\right|^{2}\right) \frac{\gamma_{S}}{\Delta^{2}}, \\
& \Gamma_{D}^{(j)}=\xi\left(|\Omega|^{2}+\left|\beta^{(j)} g\right|^{2}\right) \frac{\gamma_{D}}{\Delta^{2}} .
\end{aligned}
$$

The cavity photon annihilation operator $a=$ $\left|0^{(1)} 0^{(2)} 0^{(C)}\right\rangle\left\langle 0^{(1)} 0^{(2)} 1^{(C)}\right|$ is already normalized in our restricted basis.

The spontaneous emission decay rates for the considered states of a calcium atom are $\gamma_{S} / 2 \pi=22.3 \mathrm{MHz}$ and $\gamma_{D} / 2 \pi=1.7 \mathrm{MHz}$. Therefore, $\Gamma_{S}^{(j)} \gg \Gamma_{D}^{(j)}$ and the dominant effective spontaneous emission jump processes are described by the operators $C_{S}^{(j)}$. Consequently, according to the discussion above, the main decoherence sources are the non-dissipative dephasing processes that conserve the energy of the ion-cavity system.
The character of the decaying state, and hence the corresponding jump operator is defined by the balance between the strengths of the laser pumping $\Omega$ and the cavity coupling $\beta^{(j)} \mathrm{g}$. In the strong laser pumping case $\left(|\Omega| \gg\left|\beta^{(j)} g\right|\right)$ the non-unitary dynamics of the atomic reduced system is dominated by phase diffusion processes described by the operators $A_{11}^{(j)}$. In the weak laser pumping case $\left(|\Omega| \ll\left|\beta^{(j)} g\right|\right)$, on the contrary, the processes described by the operators $a A_{10}^{(j)}$ dominate. Moreover, as one can see from Eqs. (35) and (36), one can further modify the character of the specific atomic jump operators by changing the relative position of the ions with respect to the cavity field through the $\beta^{(j)}$ parameters.

The significance of the spontaneous emissions can be estimated by the ratio

$$
\left|\frac{\Gamma_{S}^{(j)}}{\alpha_{\text {eff }}^{(j)}}\right|=\frac{1+\left|\beta^{(j)} g / \Omega\right|^{2}}{\left|\beta^{(j)} g / \Omega\right|} \frac{\gamma_{S}}{\Delta} .
$$

For a fixed detuning $\Delta$ this ratio has its minimum value $2 \gamma_{S} / \Delta$ when $\left|\beta^{(j)} g / \Omega\right|=1$, i.e., when the couplings provided by the laser and the cavity field are equally strong. On the other hand, for fixed coupling strengths, the ratio is inversely proportional to the detuning $\Delta$. This can be exploited in order to minimize the role of the effective spontaneous decay. The cavity damping $\kappa$ is neither affected by the detuning nor the couplings.

Finally, we note that for large detunings, $\Delta \gg \gamma_{S}, \gamma_{D}$, the dimensionless prefactor $\xi \sim 1$ and the effective decay rates as well as the effective coupling terms have simplified expressions. The effective couplings are then given by $\alpha_{\text {eff }}^{(j)} \sim-\beta^{(j)} g^{*} \Omega / \Delta$, while in the limit of strong and weak laser pumpings the dominating decay rates are $\Gamma_{S}^{(j)} \sim|\Omega|^{2} \gamma_{S} / \Delta^{2}$ and $\Gamma_{S}^{(j)} \sim\left|\beta^{(j)} g\right|^{2} \gamma_{S} / \Delta^{2}$, respectively.

\section{ENVIRONMENT-INDUCED ENTANGLEMENT: RESONANT REGIME}

In this section we study, analytically and numerically, the dynamics of the entanglement between the electronic degrees of freedom of the two atoms. The generation of entanglement between the ions and its persistence at long times are, indeed, a clear manifestation of the collective (subradiant) behavior. In particular, entanglement generation is mediated by the interaction with the quantized cavity field which is initially prepared in the vacuum state. If the atomic spontaneous emission processes are negligible and we face the bare Dicke model, the dynamics can be described exactly. We compare these exact analytical results to numerical simulations including the spontaneous emission effects. The simulations were implemented by using the Monte Carlo wave function (MCWF) method [33, 34]. We begin by considering the resonant case, where the effective detuning $\delta_{\text {eff }}=0$, with $\delta_{\text {eff given by Eq. (30). }}$. 


\section{A. Analytical solution neglecting spontaneous emission}

The effective model describing the dynamics when spontaneous emissions are negligible is given by the master equation (11) with the effective Tavis-Cummings Hamiltonian (26), as described in Sec. IB The analytical solution for the atomic density matrix is given by Eqs. (12) and (15), with $\chi^{(j)} W=\alpha_{\text {eff }}^{(j)}=\beta^{(j)} g_{\text {eff }}$.

We are interested in the collective dynamics when initially one excitation is present in the atomic system and the cavity is in its vacuum state. Any initial atomic state containing one excitation can be written in terms of the superradiant and subradiant states (15)-(6) as

$$
|\psi(0)\rangle=\beta_{+}\left|\psi_{+}\right\rangle+\beta_{-}\left|\psi_{-}\right\rangle .
$$

As time passes, the collective atomic state decays via the evolution of the superradiant component,

$$
\left\langle\psi_{+} \mid \psi(t)\right\rangle=\mathcal{E}(t) \beta_{+},
$$

with $\mathcal{E}(t)$ given by Eq. (15). The subradiant component $\left\langle\psi_{-} \mid \psi(t)\right\rangle=\beta_{-}$, however, remains unchanged. Consequently, for times, such that $\kappa t \gg 1$, the atomic state will be in general a statistical mixture of the collective ground state $\left|0^{(1)} 0^{(2)}\right\rangle$ and the subradiant state $\left|\psi_{-}\right\rangle$with weights dependent on $\beta_{-}$, which in turn depends on the relative coupling strengths $r^{(j)}$.

In the following we focus on the dynamics of entanglement between the atoms. In order to quantify the stationary asymptotic entanglement of the final state we use Wootters's concurrence [35] which, for a density matrix of the form of Eq. (12), is given by

$$
C(t)=2\left|c_{10}(t) c_{01}^{*}(t)\right|,
$$

with $c_{10}(t)$ and $c_{01}(t)$ given by Eqs. (13) and (14). In general, the concurrence is zero for factorized states and unity for maximally entangled states. For $\kappa t \gg 1$ we obtain a stationary concurrence value

$$
C_{\text {stat }}=2\left|r^{(1)} r^{(2)}\right|\left|\beta_{-}\right|^{2} .
$$

As expected, the value of the stationary concurrence is directly related to the subradiant component of the initial state. If both atoms are coupled to the EM field, the stationary value of the concurrence, for any initial state with $\beta_{-} \neq 0$, will be nonzero. When the atoms are initially prepared in the superradiant state, i.e., $\beta_{-}=0$, the system approaches asymptotically the pure factorized state $\left|0^{(1)} 0^{(2)}\right\rangle$.

For the initially factorized states $\left|1^{(1)} 0^{(2)}\right\rangle$ and $\left|0^{(1)} 1^{(2)}\right\rangle$, the interaction with the environment generates entanglement in the atomic system. For these initial states the stationary concurrence takes the values $C_{\text {stat }}=$ $2\left|r^{(1)}\right|\left(1-\left|r^{(1)}\right|^{2}\right)^{3 / 2}$ and $C_{\text {stat }}=2\left|r^{(1)}\right|^{3} \sqrt{1-\left|r^{(1)}\right|^{2}}$, respectively. As we have noticed in Ref. [29], the factorized states are those that maximize the stationary concurrence for certain values of $r^{(1)}$. The maximum value of stationary concurrence, for both the two factorized initial states considered here, is $C_{\text {stat }}^{\max }=\max _{\left|r^{(1)}\right| \in[0,1]} C_{\text {stat }} \simeq$ 0.65. This value is obtained with $\left|r^{(1)}\right|=0.5$ and $\left|r^{(1)}\right| \simeq 0.87$ (i.e., $\left|r^{(2)}\right|=0.5$ ) for initial states $\left|1^{(1)} 0^{(2)}\right\rangle$ and $\left|0^{(1)} 1^{(2)}\right\rangle$, respectively.

We note in passing that when only one of the two atoms is coupled to the EM field, i.e., $r^{(1)}=0$ or $r^{(2)}=0$, the stationary concurrence is zero. In this case, indeed, the subradiant and the superradiant states coincide with states $\left|1^{(1)} 0^{(2)}\right\rangle$ and $\left|0^{(1)} 1^{(2)}\right\rangle$ as one can see from definitions (5) and (6).

From the definition of the generalized Rabi frequency given by Eq. (15), which in the resonant case reads as $\Omega_{g}=\sqrt{4\left|\beta_{T} g_{\mathrm{eff}}\right|^{2}-\kappa^{2} / 4}$, two extreme regimes can be defined. In the weak ion-cavity coupling regime, defined by $4\left|\beta_{T} g_{\text {eff }}\right| \ll \kappa$, the generalized Rabi frequency is purely imaginary. Therefore, according to Eq. (15), the Dicke model predicts a solution given by monotonic hyperbolic sine and cosine functions. The opposite limit is the strong ion-cavity coupling regime, defined by $4\left|\beta_{T} g_{\text {eff }}\right| \gg \kappa$. In this case the generalized Rabi frequency is real and the Dicke model predicts damped oscillatory dynamics.

\section{B. MCWF simulations in the presence of spontaneous emission}

In this section, we focus on the effect of the spontaneous emissions on the subradiant-state-based entanglement generation described in the previous section. We consider again as initial atomic state $|\psi(0)\rangle=\left|1^{(1)} 0^{(2)}\right\rangle$ with the cavity in the vacuum state $\left|0^{(C)}\right\rangle$. For a given value of $r^{(1)} \in[0,1]$, we choose $\beta^{(1)}$ and $\beta^{(2)}$ to be positive real numbers such that the larger of the two is always unity and the smaller one is $\min \left\{r^{(1)} / \sqrt{1-r^{(1) 2}}, \sqrt{1-r^{(1) 2}} / r^{(1)}\right\}$ [cf. definition (21)]. Now $\left|\beta_{T}\right|^{2}=\left|\beta^{(1)}\right|^{2}+\left|\beta^{(2)}\right|^{2}=$ $\min \left\{1 / r^{(1) 2}, 1 /\left(1-r^{(1) 2}\right)\right\} \in[1,2]$. The physical parameters have been chosen in accordance to the experiments of Ref. 8] and are summarized in Table [. The size of the ensemble in the MCWF simulations is $N=1000$. We are using the variant of MCWF method described in [34].

The value of the cavity coupling constant $g$ in Table I refers to the new miniature trap recently realized at the University of Sussex 36]. The reference value $\kappa_{0}$ for the cavity damping can nowadays be improved by at least one order of magnitude. Finally, the detuning $\Delta$ can be easily increased in the experiments, with respect to the reference value $\Delta_{0}$.

With the experimental parameters of Table【 the coupling strengths $\Omega$ and $g$ are of the same order. Therefore, neither the strong nor the weak laser pumping regimes, introduced in Sec. IIIC, are reached and, consequently, all the effective decay processes caused by the spontaneous emission are combinations of two different physical operations, as interpreted in Sec. IIIB

Let us denote the atomic density-matrix components as $\rho_{a b, c d} \equiv\left\langle a^{(1)} b^{(2)}|\rho| c^{(1)} d^{(2)}\right\rangle$, where $a, b, c, d=0,1$. 
TABLE I: Values of physical quantities used in the simulations. Note that the cavity coupling is here explicitly scaled by the Clebsch-Gordan factor $1 / \sqrt{3}$ and, in the text, also by the position-dependent parameters $\beta^{(j)}$.

\begin{tabular}{lcc}
\hline \hline Quantity & Symbol & Value $(2 \pi \mathrm{MHz})$ \\
\hline Laser coupling & $\Omega$ & 9.0 \\
Cavity coupling & $g$ & $6.5 / \sqrt{3}$ \\
Decay rate $4^{2} P_{1 / 2} \rightarrow 4^{2} S_{1 / 2}$ & $\gamma_{S}$ & 22.3 \\
Decay rate $4^{2} P_{1 / 2} \rightarrow 3^{2} D_{3 / 2}$ & $\gamma_{D}$ & 1.7 \\
Detuning & $\Delta_{0}$ & 20.0 \\
Cavity damping & $\kappa_{0}$ & 1.2 \\
\hline \hline
\end{tabular}

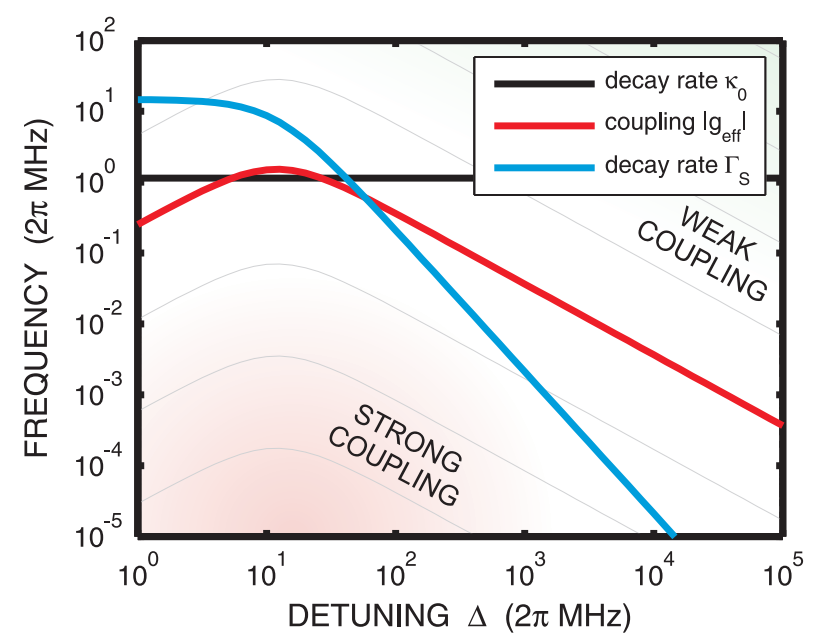

FIG. 3: (Color online) Scaling of the effective ion-cavity coupling $\left|g_{\text {eff }}\right|$ (middle line for large $\Delta$ ) and effective spontaneous emission decay rates $\Gamma_{S}^{(j)}$ (lowest line for large $\Delta$; with $\beta^{(j)}=1$ ) as a function of the detuning $\Delta$. The isocurves $4\left|\beta_{T} g_{\text {eff }}\right| / \kappa=$ const (thin lines) are parallel to the $\left|g_{\text {eff }}\right|$ curve, so that the weak ion-cavity coupling regime is in the upper right corner and the strong ion-cavity coupling regime in the lower left one. The cavity decay rate $\kappa$ (horizontal line) does not depend on the detuning. The effective spontaneous emission events are suppressed for large detunings.

The density matrix remains still in the same block form of Eq. (12) even in the presence of spontaneous emissions. The concurrence is therefore given by $C(t)=2\left|\rho_{01,10}(t)\right|$.

In the following we will examine the effect of the spontaneous emissions by comparing the concurrence as a function of time for fixed values of $4\left|\beta_{T} g_{\text {eff }}\right| / \kappa=$ $4\left|\beta_{T} \xi g \Omega / \kappa \Delta\right|$. We study large detunings $\left(\Delta \gg \gamma_{S}, \gamma_{D}\right)$, so the prefactor $\xi \sim 1$. In the examples we change $\kappa$ and $\Delta$, such that $\kappa / \kappa_{0}=0.1,0.01$ and $\Delta / \Delta_{0}=10,100,1000$, while keeping the product $\kappa \Delta$ constant. Physically, this corresponds to using different cavity qualities and detunings which, furthermore, influences the effective dynamical parameters. Larger detunings, indeed, suppress the effective spontaneous emissions in favor of the coherent dynamics, as explained in Sec. IIIC, The situation is clarified in Fig. 3 which shows the scaling of the effective coupling strength $g_{\text {eff }}$ and the dominant spontaneous emission decay rate $\Gamma_{S}^{(j)}[$ cf. Eqs. (29), (37), and (38) $]$ as functions of detuning $\Delta$. The cavity damping rate $\kappa$ is not affected by the detuning. The relative values of the three key parameters $g_{\mathrm{eff}}, \Gamma_{S}^{(j)}$, and $\kappa$ characterize the dynamical regime: (i) the ratio $\left|g_{\text {eff }}\right| / \kappa$ defines the strong and the weak ion-cavity coupling regimes and (ii) the magnitude of $\Gamma_{S}^{(j)}$ compared to $\left|g_{\text {eff }}\right|$ and $\kappa$, in turn, describes the significance of the spontaneous emission processes and tells us whether the dynamics is well described by the Dicke model or not.

\section{Weak ion-cavity coupling regime}

In this regime, the oscillatory dynamics stemming from the coherent coupling between the atoms and the cavity is heavily damped. In Fig. 4 we plot the concurrence as a function of both time and the relative coupling strength $r^{(1)}$ for $\Delta / \Delta_{0}=100$ and $\kappa / \kappa_{0}=0.1$, giving $\left|g_{\text {eff }}\right| / 2 \pi=\xi g \Omega / 2 \pi \Delta=17 \mathrm{kHz}$. All the other parameters are chosen as in Table \. We recall that initially the atomic state $|\psi(0)\rangle=\left|1^{(1)} 0^{(2)}\right\rangle$ is factorized. The initial dynamics of the concurrence shows a monotonic increase, as the superradiant component [see Eq. (40)] rapidly fades away while the subradiant component remains intact. However, because of the presence of spontaneous emission, the subradiant state is not anymore perfectly decoupled from the dynamics and, consequently, the concurrence will not reach a steady-state value.

We note that the best peak value of the concurrence $C \simeq 0.6$ is achieved for $r^{(1)} \simeq 0.55$, i.e., as expected, for an asymmetric configuration $\left(r^{(1)} \neq 1 / \sqrt{2}\right)$ of the ions with respect to the cavity field. However, this value of $r^{(1)}$ is now slightly different than the one obtained in Sec. IVA where spontaneous emissions were neglected $\left(r^{(1)}=0.5\right)$. We will further discuss this point when considering the position dependence of the jumps statistics at the end of this subsection.

In Fig. 5 we further study the effect of the spontaneous emissions in the weak ion-cavity coupling case. In this figure, we compare the predictions of the Dicke model, described in Sec. IIB with the dynamics of the ion-cavity system in the presence of the spontaneous emissions for $\Delta / \Delta_{0}=100, \kappa / \kappa_{0}=0.1$ and $\Delta / \Delta_{0}=1000, \kappa / \kappa_{0}=0.01$. The dynamics of the concurrence clearly shows that, in the first case $\left(\kappa / \kappa_{0}=0.1\right)$, the system approximates the Dicke model well while $\left|\Omega_{g}\right| t / 2 \pi<2.5$, where the generalized Rabi frequency $\left|\Omega_{g}\right|$ is given by Eq. (15). For a better cavity $\left(\kappa / \kappa_{0}=0.01\right)$, the concurrence approaches its quasi-stationary value and the system approximates the ideal Dicke dynamics for longer times, $\left|\Omega_{g}\right| t / 2 \pi<20$.

We finally look at the statistics of the quantum jumps, described by the jump operators $C_{m}^{(j)}$ in Eqs. (31)-(34). 


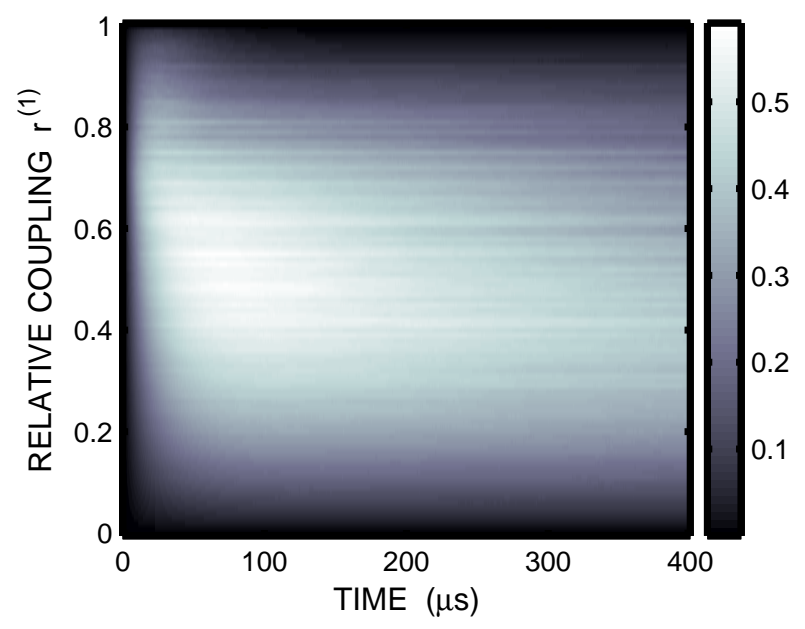

FIG. 4: (Color online) Concurrence as a function of time and the relative coupling strength $r^{(1)}$ in the weak ion-cavity coupling regime. The dynamics is initially monotonic since the existing superradiant component decays rapidly compared to other dynamical time scales. The subradiant state component decays eventually because of the atomic spontaneous emissions. The best entanglement production occurs with asymmetric couplings $\left(r^{(1)} \neq 1 / \sqrt{2}\right)$. Parameters: $\Delta / \Delta_{0}=100, \kappa / \kappa_{0}=0.1$

First of all, we note that the source states $\left|\Phi_{j}\right\rangle$ [see Eqs. (35) and (36)] of the jump operators $C_{S}^{(j)}$ and $C_{D}^{(j)}$ are identical for a given atom $j=1,2$. Therefore, the jump statistics of the two corresponding decay channels will also be the same with a branching ratio given by $\Gamma_{S}^{(j)} / \Gamma_{D}^{(j)}=\gamma_{S} / \gamma_{D} \simeq 13$. Our MCWF simulations confirm that the dominant jump processes are those corresponding to the effective spontaneous emission operators $C_{S}^{(j)}$ and the cavity photon annihilation operator $a$. In Fig. 6] we plot the average cumulative number of quantum jumps per ensemble member for the jump operators $C_{S}^{(1)}, C_{S}^{(2)}$, and $a$.

Looking at the statistics helps us to understand how the reservoir-mediated entanglement generation process depends on $r^{(1)}$. We notice that the jump statistics of processes originating from the spontaneous emissions of atoms 1 and 2 are different. This is of course due to the asymmetry in the initial condition. Since initially the excitation is present in atom 1 , the average cumulative number of jumps per ensemble member is typically greater for $C_{S}^{(1)}$ than for $C_{S}^{(2)}$. The peak in the cumulative number of jumps, for the three different jump operators considered in Fig. 6, moreover, is reached in correspondence of different values of $r^{(1)}$. This indicates that the value $r^{(1)} \simeq 0.55$, which optimizes the concurrence generation (see Fig. 4), corresponds to a compromise between the different $r^{(1)}$-dependent jump statistics. In particular, the deviation from the optimal value in the absence of spontaneous emission $\left(r^{(1)}=0.5\right)$ might be

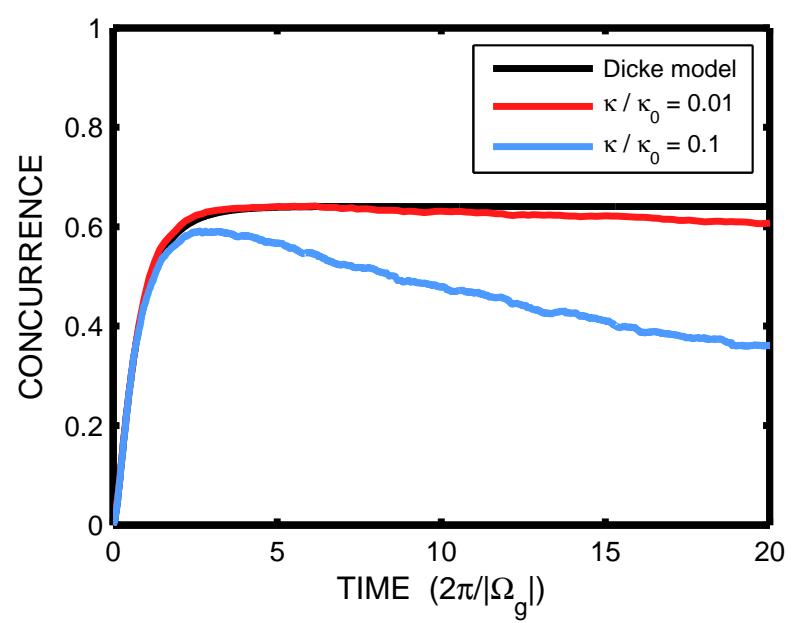

FIG. 5: (Color online) Dynamics of the concurrence in the weak ion-cavity coupling regime for $r^{(1)}=0.55$. In the Dicke model with cavity losses (highest line) a stationary value of the concurrence is reached as the superradiant component is over-damped. Parameters: $\Delta / \Delta_{0}=100, \kappa / \kappa_{0}=0.1$ (lowest line, $2 \pi /\left|\Omega_{g}\right|=23 \mu \mathrm{s}$ ); $\Delta / \Delta_{0}=1000, \kappa / \kappa_{0}=0.01$ (middle line, $\left.2 \pi /\left|\Omega_{g}\right|=230 \mu \mathrm{s}\right)$.

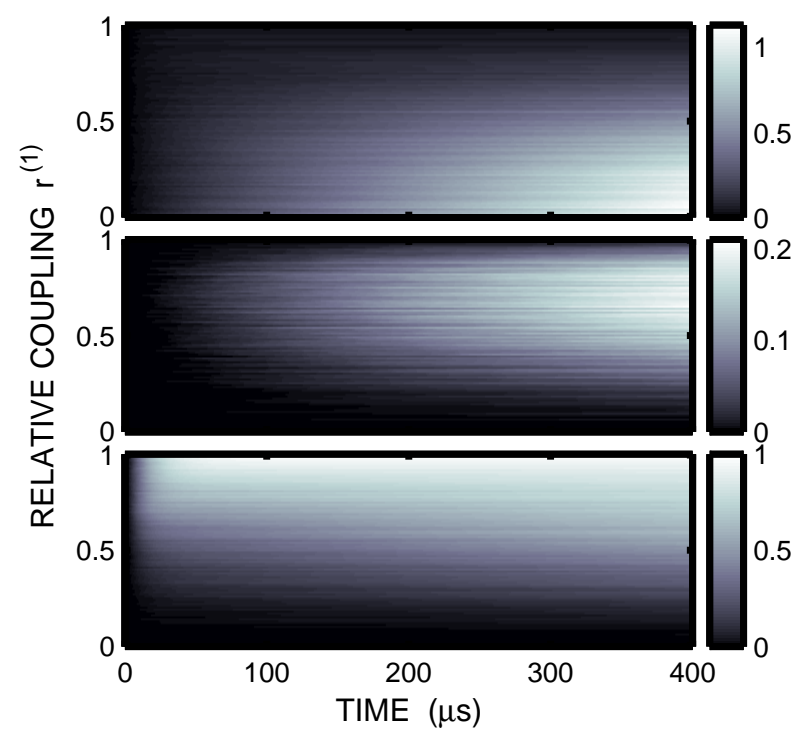

FIG. 6: (Color online) Average cumulative number of quantum jumps per ensemble member for each decay channel in the weak ion-cavity coupling regime. From above: $C_{S}^{(1)}, C_{S}^{(2)}$, and $a$. Parameters: $\Delta / \Delta_{0}=100, \kappa / \kappa_{0}=0.1$.

due to the fact that the number of $C_{S}^{(1)}$-jumps increases for decreasing values of $r^{(1)}$. 


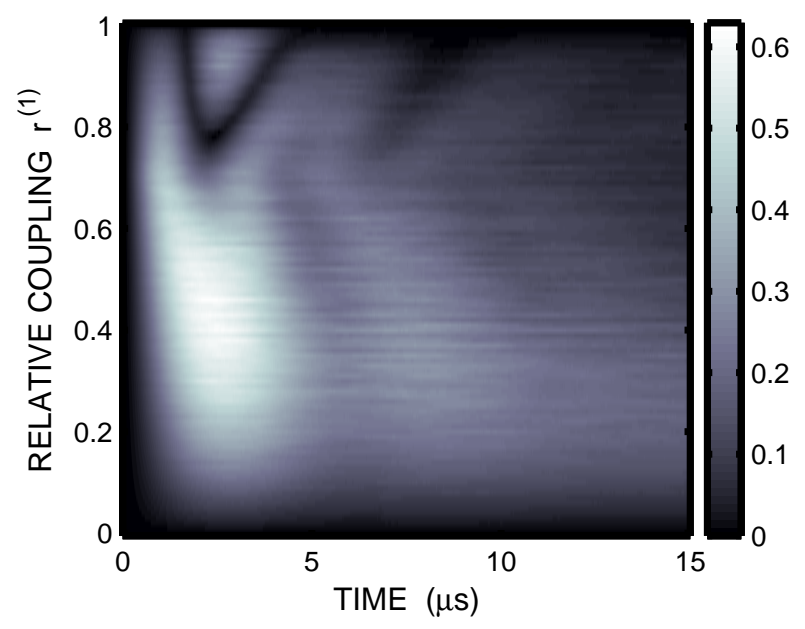

FIG. 7: (Color online) Concurrence as a function of time for different values of the relative coupling strength $r^{(1)}$ in the strong ion-cavity coupling regime. Oscillations appear because of a relative phase evolution between the superradiant and subradiant states. Parameters: $\Delta / \Delta_{0}=10$ and $\kappa / \kappa_{0}=$ 0.1 .

\section{Strong ion-cavity coupling regime}

In the strong ion-cavity coupling regime, the cavity damping is slow compared to the coherent dynamics. Therefore, a slowly damped oscillatory behavior of the concurrence is expected. In Fig. 7 we plot the concurrence as a function of both time and the relative coupling strength $r^{(1)}$ for $\Delta / \Delta_{0}=10$ and $\kappa / \kappa_{0}=0.1$, giving $\left|g_{\text {eff }}\right| / 2 \pi=\xi g \Omega / 2 \pi \Delta=170 \mathrm{kHz}$. All the other parameters are chosen as in Table प. Note that the ratio $\left|g_{\text {eff }}\right| / \kappa$ is now one order of magnitude bigger than in Sec. IVB 1. The dynamics has an oscillatory character, since the superradiant component survives much longer than in the weak ion-cavity coupling regime. However, due to the presence of the spontaneous emissions the concurrence does not reach a steady-state value in this regime either.

The best peak value of the concurrence, $C \simeq 0.6$, is now obtained for $r^{(1)} \simeq 0.46$. In Fig. 8 we choose this value of $r^{(1)}$ and we compare the dynamics of the single-mode Dicke model with cavity losses to the dynamics of the ion-cavity system in the presence of effective spontaneous emissions for the cases of $\Delta / \Delta_{0}=10$ with $\kappa / \kappa_{0}=0.1$, and $\Delta / \Delta_{0}=100$ with $\kappa / \kappa_{0}=0.01$. In the second case, i.e., for a better quality factor, the system approximates the Dicke model for longer time scales, as one would expect. In this case one can clearly observe the damped Rabi oscillation at the generalized Rabi frequency given by Eq. (15).

It is worth noticing that, in the strong ion-cavity coupling regime, the laser-mediated interaction with the cavity vacuum allows us to generate a highly entangled state of the two ions, as one can see in Fig. 8 . In particular,

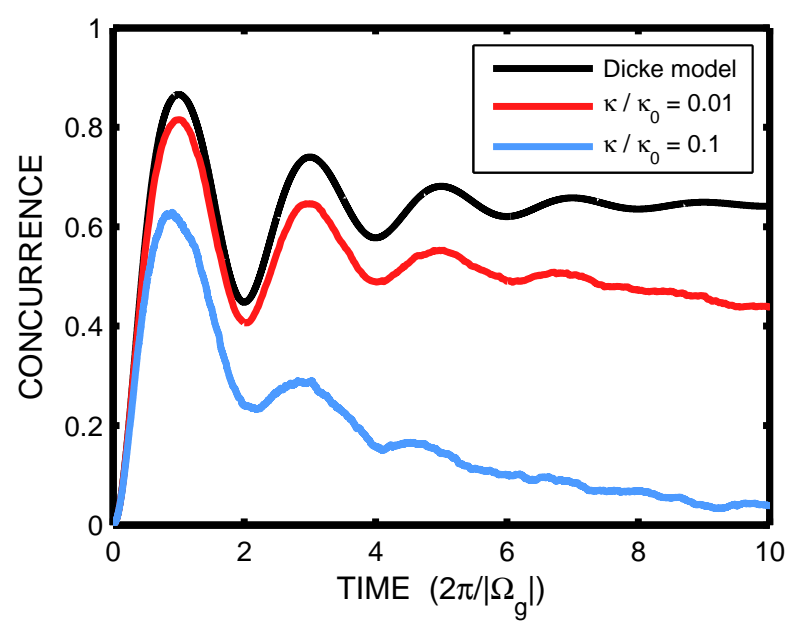

FIG. 8: (Color online) Time evolution of the concurrence in the strong ion-cavity coupling regime with relative coupling strength $r^{(1)}=0.46$. For the Dicke model with cavity losses (highest line), the concurrence approaches a constant stationary value after strong oscillations caused by the slowly decaying superradiant component. Parameters: $\Delta / \Delta_{0}=10, \kappa / \kappa_{0}=0.1$ (lowest line, $2 \pi /\left|\Omega_{g}\right|=2.7 \mu \mathrm{s}$ ); $\Delta / \Delta_{0}=100, \kappa / \kappa_{0}=0.01$ (middle line, $2 \pi /\left|\Omega_{g}\right|=27 \mu \mathrm{s}$ ).

for $\Delta / \Delta_{0}=100$ with $\kappa / \kappa_{0}=0.01$, using a laser pulse of duration $t \simeq 2 \pi /\left|\Omega_{g}\right|$, the generated state is close to a maximally entangled Bell state.

\section{ENVIRONMENT-INDUCED ENTANGLEMENT: DISPERSIVE REGIME}

In the previous section we have seen that by placing the ions properly, i.e., by adjusting the relative coupling strength $r^{(1)}$, it is possible to optimize the reservoirmediated entanglement generation. The examples discussed above deal with the resonant effective model, which is defined by the condition $\delta_{\text {eff }}=0$, which in turn corresponds to a physical detuning $\delta_{L}=\xi\left[\Omega^{2}-\right.$ $\left.\left(\beta_{T} g\right)^{2}\right] / \Delta$ [cf. Eq. [301)]. We have seen that the highest value of the concurrence is obtained in the strong ioncavity coupling regime.

In Ref. [37], however, the single-mode Dicke model with cavity losses is studied in the dispersive regime, showing that a high degree of entanglement can be obtained also in the weak ion-cavity coupling regime. For this reason we now look at the off-resonant entanglement generation process in the ion-cavity QED, i.e., we consider the case in which $\delta_{\text {eff }} \neq 0$. In the dispersive regime, the relative position of the ions does not play an essential role and in fact one shows that the optimal value of $r^{(1)}$ is obtained for equal coupling of the two ions, i.e., $r^{(1)}=r^{(2)}=1 / \sqrt{2}$ [37].

We consider once more the initial atomic state $|\psi(0)\rangle=$ $\left|1^{(1)} 0^{(2)}\right\rangle$ combined with the cavity in the vacuum state 


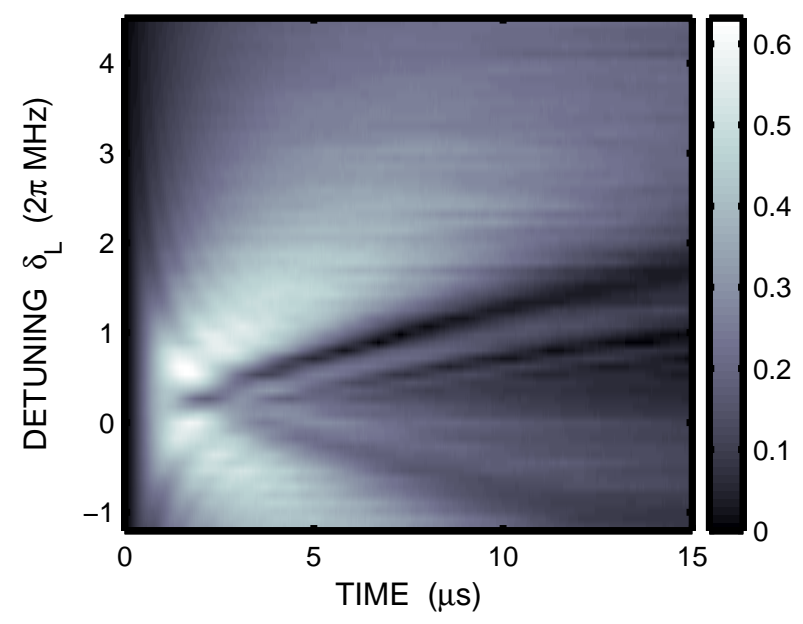

FIG. 9: (Color online) Concurrence as a function of time for different values of the detuning $\delta_{L}$ for a homogeneously coupled case $\left(r^{(1)}=r^{(2)}=1 / \sqrt{2}\right)$. The entanglement generation slows down when passing into the dispersive regime. The resonance is dislocated from the origin because of the Stark shifts. Parameters: $\Delta / \Delta_{0}=10$ and $\kappa / \kappa_{0}=0.1$.

$\left|0^{(C)}\right\rangle$. We set $r^{(1)}=r^{(2)}=1 / \sqrt{2}$ (by choosing $\max -$ imally strong cavity-driven couplings $\beta^{(1)}=\beta^{(2)}=1$ ), $\Delta / \Delta_{0}=10$, and $\kappa / \kappa_{0}=0.1$, corresponding to the weak ion-cavity coupling regime of Sec.IVB 1. We now look at the time evolution of the concurrence for different values of the laser detuning $\delta_{L}$. Figure 9 shows the concurrence as a function of both time and detuning $\delta_{L}$. One can see clearly that the Stark shift terms appearing in the effective Hamiltonian of Eq. (20) relocate the resonance condition from the origin to $\delta_{L} / 2 \pi=\xi\left[\Omega^{2}-\left(\beta_{T} g\right)^{2}\right] / 2 \pi \Delta=$ $120 \mathrm{kHz}$. Figure 9 also shows that selecting the detuning $\delta_{L}$ further away from the resonance produces higher values of concurrence. In particular, with the chosen parameters the maximum value of concurrence $C \simeq 0.62$ is obtained with $\delta_{L} / 2 \pi \simeq 600 \mathrm{kHz}$.

As demonstrated in Ref. 37], increasing the detuning $\left|\delta_{\mathrm{eff}}\right|$ correspondingly increases the time it takes for the concurrence to reach its peak value. The longer is the entanglement generation time, however, the stronger is the effect of the spontaneous emissions. In other words, the achieved gain in the entanglement generation obtained by increasing the effective detuning is quickly suppressed due to the spontaneous decay, as the overall time of the entanglement generation process increases. The maximum value of entanglement achievable in the dispersive regime is therefore determined by the interplay between these two effects.

It is worth noticing that going from the resonant into the dispersive regime changes the character of the generated entangled state as well. To illustrate this point, we plot in Figs. 10 and 11 the populations and coherences, respectively, of the reduced atomic density matrix versus time and detuning $\delta_{L}$. These plots confirm the

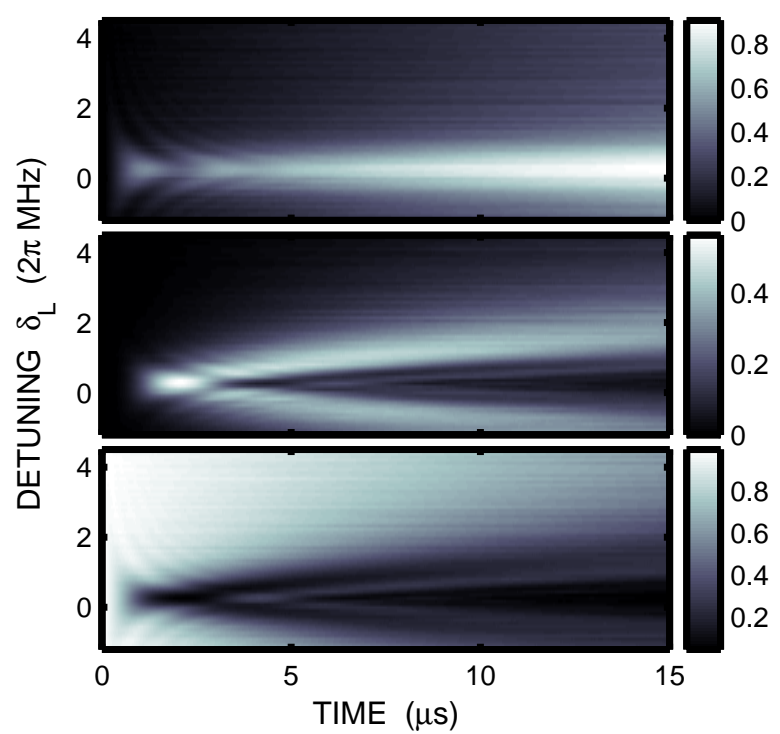

FIG. 10: (Color online) Populations of the atomic states $\rho_{00,00}, \rho_{01,01}$, and $\rho_{10,10}$ (from above) as a function of time for different values of detuning $\delta_{L}$. Parameters are as in Fig. 9.

increase in the entanglement generation time when going deeper and deeper into the dispersive regime $\left(\left|\delta_{\text {eff }}\right|>0\right)$. If we then focus on the dynamics of the coherences and, in particular, on the real and imaginary parts of the only nonzero off-diagonal element $\rho_{01,10}$, we see that on resonance the imaginary part vanishes in accordance with the predictions of Sec. IV] Therefore, in the resonant regime the generated entangled state approximates the subradiant state. On the other hand, in the dispersive regime $\operatorname{Re}\left[\rho_{01,10}\right] \simeq 0$ and $\operatorname{Im}\left[\rho_{01,10}\right] \neq 0$. Indeed, in the absence of the spontaneous emissions, the generated state in the dispersive regime would be $\left(\left|1^{(1)} 0^{(2)}\right\rangle \pm i\left|0^{(1)} 1^{(2)}\right\rangle\right) / \sqrt{2}$ (positive sign for negative $\delta_{\text {eff }}$ and vice versa).

\section{SUMMARY AND CONCLUSIONS}

In this paper we have investigated how the singlemode Dicke model can be realized under experimentally feasible conditions using two trapped ${ }^{40} \mathrm{Ca}^{+}$ions inside a high-finesse optical cavity. We have taken into account the spontaneous emissions of the ions as well as the damping of the electromagnetic field inside the cavity. In particular, we have derived an effective two-level description of the three-level ions interacting with the cavity mode.

We have shown that under suitable conditions the two ions indeed behave collectively, with a coherent dynamical evolution well described by the Dicke model: two effective two-level systems exchanging an excitation with an effective one-dimensional cavity mode. The presence of decohering processes, such as the atomic spontaneous emission or the cavity field damping, modifies this ideal 


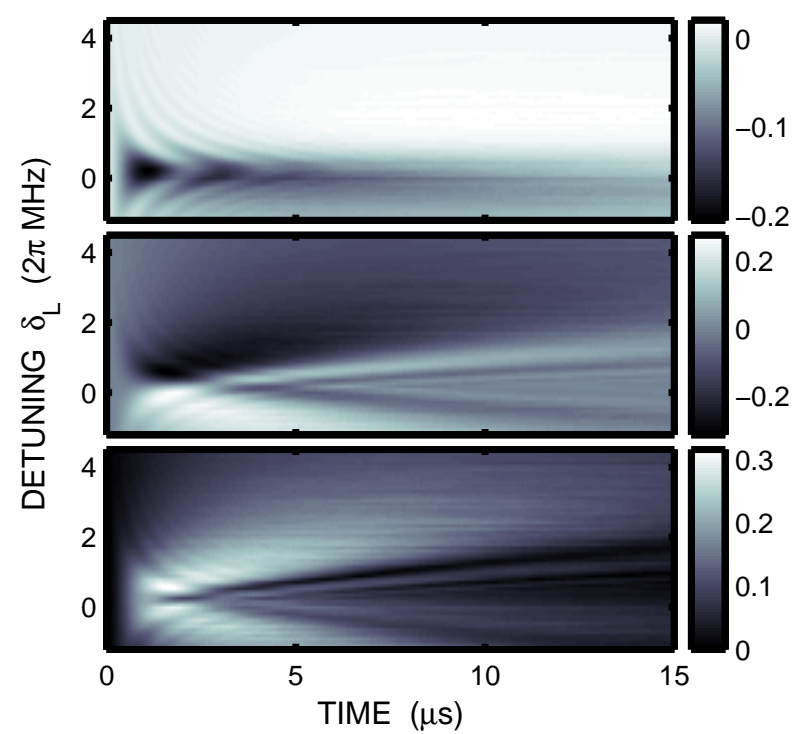

FIG. 11: (Color online) Dynamics of $\operatorname{Re}\left[\rho_{01,10}\right], \operatorname{Im}\left[\rho_{01,10}\right]$, and $\left|\rho_{01,10}\right|$ (from above) as a function of time for different values of detuning $\delta_{L}$ (observed concurrence is given by $C=$ $\left.2\left|\rho_{01,10}\right|\right)$. Parameters are as in Fig. 9

picture. However, in the effective model, the spontaneous emission decay rates are proportional to $1 / \Delta^{2}$ whereas the ion-cavity couplings scale as $1 / \Delta$, where $\Delta$ is the detuning of the physical cavity frequency from the electronic transition that it is driving. This difference in the scaling can be exploited in order to partly suppress the destructive effect of the atomic spontaneous emissions.

We have identified the generation of entanglement as a fingerprint of the cooperative atomic behavior and analyzed this process in detail. In particular, we have proven that it is possible to enhance the entanglement generation process by positioning the ions appropriately at different locations with respect to the standing mode of the electromagnetic field inside the cavity. In the resonant case, where the two-level systems and the cavity mode have the same frequency, we have shown that asymmetric coupling with the cavity mode produces the highest degree of entanglement, even in presence of spontaneous emissions. We have studied both the weak and the strong ion-cavity coupling regimes, defined by the strength of the ion-cavity excitation exchange compared to the cavity field damping rate, and found out the optimal conditions for entanglement generation in both cases.

Another possibility to optimize the entanglement generation is to go to the dispersive regime in the ion-cavity coupling by using an off-resonant Raman transition. The maximum degree of entanglement in the dispersive and in the resonant regimes, for realistic values of the parameters, is similar. Our results indicate, however, that the character of the generated entangled state in the dispersive regime changes compared to the resonant case.

Our experimental proposal is based on existing tech- nology used in the context of ion-cavity QED experiments [8, 9, 30]. In order to detect the generated entanglement, the state tomography of the atomic systems is needed. In recent years, this has been routinely performed in similar trapped-ion systems, e.g., in the context of quantum computation and measuring the quality of quantum gates [31]. Therefore, we expect our proposal to be within the reach of the experimental community.

\section{Acknowledgments}

The authors thank K.-A. Suominen for useful discussions. S.M. thanks B. Garraway, M. Keller, and W. Lange for discussions on the experimental implementation of the ion-cavity QED setup and for the kind hospitality at the University of Sussex. This work was supported by the National Graduate School of Modern Optics and Photonics and the Magnus Ehrnrooth Foundation (K.H.), the Academy of Finland (Projects No. 108699, No. 115682, No. 115982, and No. 8125004), the Väisälä Foundation, and the Turku Collegium of Science and Medicine (S.M.).

\section{APPENDIX A}

In this appendix we show how the master equation (16) for $\Lambda$-coupled three-level atoms and a cavity photon is transformed into an effective two-level master equation (23) by adiabatic elimination of the excited atomic states $\left\{\left|P^{(j)}\right\rangle\right\}$. Especially, the elimination transforms the jump operators related to the spontaneous emissions into the form given by Eqs. (24) and (25). For the sake of generality, our treatment here is valid for $N$ atoms and we allow each ion $j$ to be irradiated by a separate pump laser with frequency $\omega_{L}+\delta_{L}^{(j)}$ and coupling strength $g_{L}^{(j)}$.

Passing into interaction picture $\varrho \mapsto \tilde{\varrho}=e^{i K t} \varrho e^{-i K t}$ with respect to

$$
K=\omega_{C}\left(a^{\dagger} a+\frac{1}{2}\right)+\sum_{j} \sum_{l=S, P, D} \omega_{l} A_{l l}^{(j)}-\Delta \sum_{j} A_{P P}^{(j)}
$$

transforms the operators as

$$
\begin{aligned}
a & \mapsto \tilde{a}=e^{-i \omega_{C} t} a, \\
A_{P S}^{(j)} & \mapsto \tilde{A}_{P S}^{(j)}=e^{+i \omega_{L} t} A_{P S}^{(j)}, \\
A_{P D}^{(j)} & \mapsto \tilde{A}_{P D}^{(j)}=e^{+i \omega_{C} t} A_{P D}^{(j)}, \\
A_{D S}^{(j)} & \mapsto \tilde{A}_{D S}^{(j)}=e^{+i\left(\omega_{L}+\omega_{C}\right) t} A_{D S}^{(j)}, \\
A_{l l}^{(j)} & \mapsto \tilde{A}_{l l}^{(j)}=A_{l l}^{(j)}, \quad l=S, P, D,
\end{aligned}
$$

for all atoms $j$, and the Hamiltonian (17) becomes

$$
\tilde{H}=\Delta \sum_{j} A_{P P}^{(j)}+\sum_{j}\left(g_{L}^{(j)} e^{-i \delta_{L}^{(j)} t} A_{P S}^{(j)}+g_{C}^{(j)} a A_{P D}^{(j)}+H . c .\right) .
$$


The dissipator part of the master equation (16) is invariant under this transformation.

Let us define a projection $\mathcal{P}$ to a subspace spanned by the to-be-eliminated atomic states $\left\{\left|P^{(j)}\right\rangle\right\}$, and another projection $\mathcal{Q}$ to the complementary subspace by

$$
\begin{aligned}
& \mathcal{P} \equiv \sum_{j} A_{P P}^{(j)} \otimes \mathbf{1}_{\mathrm{cav}} \\
& \mathcal{Q} \equiv \mathbf{1}-\mathcal{P}=\sum_{j}\left(A_{S S}^{(j)}+A_{D D}^{(j)}\right) \otimes \mathbf{1}_{\mathrm{cav}}
\end{aligned}
$$

Correspondingly, the density matrix divides into four sections

$$
\begin{aligned}
\tilde{\varrho} & =\mathcal{Q} \varrho \mathcal{Q}+\mathcal{Q} \varrho \mathcal{P}+\mathcal{P} \varrho \mathcal{Q}+\mathcal{P} \varrho \mathcal{P} \\
& \equiv \varrho_{Q Q}+\varrho_{Q P}+\varrho_{P Q}+\varrho_{P P} .
\end{aligned}
$$

The Hamiltonian is similarly divided in parts

$$
\begin{aligned}
H_{P P} & \equiv \mathcal{P} \tilde{H} \mathcal{P}=\Delta \sum_{j} A_{P P}^{(j)}, \\
H_{P Q} & =H_{Q P}^{\dagger} \equiv \mathcal{P} \tilde{H} \mathcal{Q} \\
& =\sum_{j}\left(g_{L}^{(j)} e^{-i \delta_{L}^{(j)} t} A_{P S}^{(j)}+g_{C}^{(j)} a A_{P D}^{(j)}\right), \\
H_{Q Q} & \equiv \mathcal{Q H} \mathcal{Q}=0 .
\end{aligned}
$$

We proceed to deriving an effective master equation for $\varrho_{Q Q}$, which describes the dynamics of a collection of effective two-level atoms and a cavity mode. Applying Eqs. (A10)- (A13) to master equation (16) gives

$$
\begin{aligned}
\dot{\varrho}_{Q Q}= & -i H_{Q P} \varrho_{P Q}+i \varrho_{Q P} H_{P Q}-\frac{\kappa}{2}\left(\left\{a^{\dagger} a, \varrho_{Q Q}\right\}\right. \\
& \left.-2 a \varrho_{Q Q} a^{\dagger}\right)+\gamma_{S} \sum_{j} A_{S P}^{(j)} \varrho_{P P} A_{P S}^{(j)} \\
& +\gamma_{D} \sum_{j} A_{D P}^{(j)} \varrho_{P P} A_{P D}^{(j)}, \\
\varrho_{Q P}= & \dot{\varrho}_{P Q}^{\dagger}=-\left(\frac{\gamma_{S}+\gamma_{D}}{2}-i \Delta\right) \varrho_{Q P}+i \varrho_{Q Q} H_{Q P} \\
& -i H_{Q P} \varrho_{P P}-\frac{\kappa}{2}\left(\left\{a^{\dagger} a, \varrho_{Q P}\right\}-2 a \varrho_{Q P} a^{\dagger}\right), \\
\varrho_{P P}= & -\left(\gamma_{S}+\gamma_{D}\right) \varrho_{P P}-i H_{P Q} \varrho_{Q P}+i \varrho_{P Q} H_{Q P} \\
& -\frac{\kappa}{2}\left(\left\{a^{\dagger} a, \varrho_{P P}\right\}-2 a \varrho_{P P} a^{\dagger}\right) .
\end{aligned}
$$

Setting $\dot{\varrho}_{Q P}=\dot{\varrho}_{P Q}=0$, assuming $\varrho_{Q Q} \gg \varrho_{P P}$, and neglecting the cavity damping in Eq. A15 gives an approximation

$$
\varrho_{Q P}=\varrho_{P Q}^{\dagger} \simeq \frac{-\Delta+i\left(\gamma_{S}+\gamma_{D}\right) / 2}{\Delta^{2}+\left(\gamma_{S}+\gamma_{D}\right)^{2} / 4} \varrho_{Q Q} H_{Q P}
$$

Similarly, setting $\dot{\varrho}_{P P}=0$ in Eq. A16 and using the above approximations for $\varrho_{Q P}$ and $\varrho_{P Q}$ gives

$$
\begin{aligned}
\varrho_{P P} & \simeq-\frac{i}{\gamma_{S}+\gamma_{D}}\left(H_{P Q} \varrho_{Q P}-\varrho_{P Q} H_{Q P}\right) \\
& \simeq \frac{1}{\Delta^{2}+\left(\gamma_{S}+\gamma_{D}\right)^{2} / 4} H_{P Q} \varrho_{Q Q} H_{Q P} .
\end{aligned}
$$

Finally, by inserting Eqs. A17 and A18 into Eq. (A14), we arrive at an approximated master equation 


$$
\begin{aligned}
\dot{\varrho}_{Q Q} \simeq & -i\left[\frac{-\Delta}{\Delta^{2}+\left(\gamma_{S}+\gamma_{D}\right)^{2} / 4} H_{Q P} H_{P Q}, \varrho_{Q Q}\right]-\frac{\kappa}{2}\left(\left\{a^{\dagger} a, \varrho_{Q Q}\right\}-2 a \varrho_{Q Q} a^{\dagger}\right) \\
& -\sum_{j} \frac{1}{2} \frac{\gamma_{S}}{\Delta^{2}+\left(\gamma_{S}+\gamma_{D}\right)^{2} / 4}\left[\left\{\left(H_{Q P} A_{P S}^{(j)}\right)\left(A_{S P}^{(j)} H_{P Q}\right), \varrho_{Q Q}\right\}-2\left(A_{S P}^{(j)} H_{P Q}\right) \varrho_{Q Q}\left(H_{Q P} A_{P S}^{(j)}\right)\right] \\
& -\sum_{j} \frac{1}{2} \frac{\gamma_{D}}{\Delta^{2}+\left(\gamma_{S}+\gamma_{D}\right)^{2} / 4}\left[\left\{\left(H_{Q P} A_{P D}^{(j)}\right)\left(A_{D P}^{(j)} H_{P Q}\right), \varrho_{Q Q}\right\}-2\left(A_{D P}^{(j)} H_{P Q}\right) \varrho_{Q Q}\left(H_{Q P} A_{P D}^{(j)}\right)\right] \\
\equiv & -i\left[H_{\mathrm{eff}}, \varrho_{Q Q}\right]-\frac{\kappa}{2}\left(\left\{a^{\dagger} a, \varrho_{Q Q}\right\}-2 a \varrho_{Q Q} a^{\dagger}\right)-\sum_{j} \sum_{m=S, D} \frac{\Gamma_{m}^{(j)}}{2}\left[\left\{C_{m}^{(j) \dagger} C_{m}^{(j)}, \varrho_{Q Q}\right\}-2 C_{m}^{(j)} \varrho_{Q Q} C_{m}^{(j) \dagger}\right],
\end{aligned}
$$

which has the form of the master equation (23). We can now recognize the effective Hamiltonian [cf. Eq. (20)] as

$$
\begin{aligned}
H_{\mathrm{eff}} \equiv & -\frac{\Delta}{\Delta^{2}+\left(\frac{\gamma_{S}+\gamma_{D}}{2}\right)^{2}} H_{Q P} H_{P Q} \\
= & -\xi \sum_{j}\left[\left(e^{-i \delta_{L}^{(j)} t} \frac{g_{C}^{(j) *} g_{L}^{(j)}}{\Delta} a^{\dagger} A_{D S}^{(j)}+\text { h.c. }\right)\right. \\
& \left.+\frac{\left|g_{L}^{(j)}\right|^{2}}{\Delta} A_{S S}^{(j)}+\frac{\left|g_{C}^{(j)}\right|^{2}}{\Delta} a^{\dagger} a A_{D D}^{(j)}\right],
\end{aligned}
$$

the effective spontaneous emission jump operators [cf. Eqs. (24) and (25)] as

$$
\begin{aligned}
& C_{S}^{(j)}=A_{S P}^{(j)} H_{P Q}=g_{L}^{(j)} e^{-i \delta_{L}^{(j)} t} A_{S S}^{(j)}+g_{C}^{(j)} a A_{S D}^{(j)}, \\
& C_{D}^{(j)}=A_{D P}^{(j)} H_{P Q}=g_{L}^{(j)} e^{-i \delta_{L}^{(j)} t} A_{D S}^{(j)}+g_{C}^{(j)} a A_{D D}^{(j)},
\end{aligned}
$$

(N.B., the operators are unique up to a global phase factor $e^{i \theta_{m}^{(j)}}$, where $\theta_{m}^{(j)} \in \mathbb{R}$ ), and the corresponding decay rates as

$$
\begin{aligned}
\Gamma_{S}^{(j)} & =\frac{\gamma_{S}}{\Delta^{2}+\left(\gamma_{S}+\gamma_{D}\right)^{2} / 4}=\xi \frac{\gamma_{S}}{\Delta^{2}}, \\
\Gamma_{D}^{(j)} & =\frac{\gamma_{D}}{\Delta^{2}+\left(\gamma_{S}+\gamma_{D}\right)^{2} / 4}=\xi \frac{\gamma_{D}}{\Delta^{2}} .
\end{aligned}
$$

In the above equations the dimensionless prefactor $\xi$ is as defined in Eq. (22).

\section{APPENDIX B}

In this appendix, we review how the effective two-level Hamiltonian (20) with Stark shifts is matched exactly with the Tavis-Cummings Hamiltonian (10) by passing into a rotating frame. Moreover, we show how to exploit the same phase transformation in order to simplify the propagator for the numerical simulations. As in Appendix $\mathrm{A}$ we assume each atom $j=1, \ldots, N$ to be addressed by its own pump laser with independent detunings $\delta_{L}^{(j)}$ and couplings $g_{L}^{(j)}$.

We will restrict ourselves to the subspace with at most one excitation. We introduce the following notation for the atomic states:

$$
\begin{aligned}
\left|\varphi_{0}\right\rangle & =\left|0^{(1)} \cdots 0^{(N)}\right\rangle \\
\left|\varphi_{1}^{(j)}\right\rangle & =\left|0^{(1)} \cdots 1^{(j)} \cdots 0^{(N)}\right\rangle
\end{aligned}
$$

so that the Hamiltonian (20) reads

$$
\begin{aligned}
H= & \sum_{j}\left[\left(e^{-i \delta_{L}^{(j)} t} \lambda^{(j)}\left|\varphi_{0} 1^{(C)}\right\rangle\left\langle\varphi_{1}^{(j)} 0^{(C)}\right|+\text { H.c. }\right)\right. \\
& \left.+S^{(j)}\left|\varphi_{1}^{(j)} 0^{(C)}\right\rangle\left\langle\varphi_{1}^{(j)} 0^{(C)}\right|\right] \\
& +S^{(C)}\left|\varphi_{0} 1^{(C)}\right\rangle\left\langle\varphi_{0} 1^{(C)}\right|,
\end{aligned}
$$

with Stark shifts $S^{(C)}=-\xi \sum_{j}\left|g_{C}^{(j)}\right|^{2} / \Delta$ and $S^{(j)}=$ $-\xi\left|g_{L}^{(j)}\right|^{2} / \Delta$, and effective ion-cavity couplings $\lambda^{(j)}=$ $-\xi g_{C}^{(j) *} g_{L}^{(j)} / \Delta$. The time dependence of the coupling terms will be eliminated by a phase rotation of the basis vectors

$$
\begin{aligned}
\left|\varphi_{0} 0^{(C)}\right\rangle & \mapsto e^{i \mu t}\left|\varphi_{0} 0^{(C)}\right\rangle, \\
\left|\varphi_{0} 1^{(C)}\right\rangle & \mapsto e^{i \nu t}\left|\varphi_{0} 1^{(C)}\right\rangle, \\
\left|\varphi_{1}^{(j)} 0^{(C)}\right\rangle & \mapsto e^{i\left(\delta_{L}^{(j)}+\nu\right) t}\left|\varphi_{1}^{(j)} 0^{(C)}\right\rangle,
\end{aligned}
$$

where $\mu, \nu \in \mathbb{R}$ are up to now free parameters. The Hamiltonian transforms accordingly into

$$
\begin{aligned}
H \mapsto H^{\prime}= & \sum_{j}\left[\left(\lambda^{(j)}\left|\varphi_{0} 1^{(C)}\right\rangle\left\langle\varphi_{1}^{(j)} 0^{(C)}\right|+H . c .\right)\right. \\
& \left.+\left(S^{(j)}+\delta_{L}^{(j)}+\nu\right)\left|\varphi_{1}^{(j)} 0^{(C)}\right\rangle\left\langle\varphi_{1}^{(j)} 0^{(C)}\right|\right] \\
& +\left(S^{(C)}+\nu\right)\left|\varphi_{0} 1^{(C)}\right\rangle\left\langle\varphi_{0} 1^{(C)}\right| \\
& +\mu\left|\varphi_{0} 0^{(C)}\right\rangle\left\langle\varphi_{0} 0^{(C)}\right|
\end{aligned}
$$

The requirement of a full compatibility with the TavisCummings Hamiltonian (1) within our restricted Hilbert space demands that $\mu=\mu(\nu)=\left(S^{(C)}+\nu\right) / 3$. Consequently, the effective Dicke model parameters are identi- 
fied as [cf. Eqs. (27)-(29)]

$$
\begin{aligned}
\omega_{C}^{\mathrm{eff}} & =\frac{2}{3} S^{(C)}+\frac{2}{3} \nu, \\
\omega_{A}^{\mathrm{eff}(j)} & =\delta_{L}^{(j)}+S^{(j)}-\frac{1}{3} S^{(C)}+\frac{2}{3} \nu, \\
\alpha_{\mathrm{eff}}^{(j)} & =\lambda^{(j)} .
\end{aligned}
$$

Moreover, the detunings are [cf. Eq. [30)]

$$
\delta_{\text {eff }}^{(j)}=\omega_{A}^{\mathrm{eff}(j)}-\omega_{C}^{\mathrm{eff}}=\delta_{L}^{(j)}+S^{(j)}-S^{(C)} .
$$

This means that the resonance condition of the Dicke model (cf. Sec.IV) is achieved with laser detunings $\delta_{L}^{(j)}=$ $S^{(C)}-S^{(j)}$, while in the dispersive regime (cf. Sec. V) $\delta_{L}^{(j)} \neq S^{(C)}-S^{(j)}$. As a conclusion, having each (identical) atom driven by their own pump laser allows us to simulate the inhomogeneous Dicke model (1) with independent two-level transition frequencies $\omega_{A}^{(j)}$ and couplings $\alpha^{(j)}$. On the other hand, if one has only a single laser driving all of the atoms, the transition frequencies are the same for every atom $j$, but the coupling constants $\alpha^{(j)}$ remain independent because of the positiondependent cavity couplings $g_{C}^{(j)}$.

Another aspect of the performed phase transformation is provided by how they affect the dissipator part of the master equation (23). Within our restricted Hilbert space, the jump operators transform now as

$$
\begin{aligned}
C_{S}^{(j)} & \mapsto e^{-i\left(\delta_{L}^{(j)}+\nu\right) t}\left|\varphi_{1}^{(j)} 0^{(C)}\right\rangle\left\langle\Phi_{j}\right|, \\
C_{D}^{(j)} & \mapsto e^{-i\left(\delta_{L}^{(j)}+\nu\right) t}\left|\varphi_{0} 0^{(C)}\right\rangle\left\langle\Phi_{j}\right|, \\
a & \mapsto e^{-i[\mu(\nu)-\nu] t}\left|\varphi_{0} 0^{(C)}\right\rangle\left\langle\varphi_{0} 1^{(C)}\right|
\end{aligned}
$$

(global phase factors can be discarded immediately), where the decaying (un-normalized) states are

$$
\left|\Phi_{j}\right\rangle=e^{-i \nu t} g_{L}^{(j) *}\left|\varphi_{1}^{(j)} 0^{(C)}\right\rangle+g_{C}^{(j) *}\left|\varphi_{0} 1^{(C)}\right\rangle .
$$

In the numerical MCWF simulations, the dynamics is generated by a non-Hermitian Monte Carlo Hamiltonian $H_{\mathrm{MC}}=H-\frac{i}{2} \sum_{m} \Delta_{m} J_{m}^{\dagger} J_{m}$, where $H$ is the Hermitian Hamiltonian of the master equation, and $J_{m}$ and $\Delta_{m}$ are all the jump operators and corresponding decay rates picked up from the dissipator part of the master equation. From the practical point of view, it is advantageous to have a time-independent $H_{\mathrm{MC}}$, since then the Dyson series of the propagator simplifies into exponential form $U\left(t, t_{0}\right)=\exp \left[-i H_{\mathrm{MC}}\left(t-t_{0}\right)\right]$. This is now achieved simply by choosing $\nu=0$, and hence the phase transformation is unique.
[1] R. H. Dicke, Phys. Rev. 93, 99 (1954).

[2] M. Gross and S. Haroche, Phys. Rep. 93, 301 (1982).

[3] Z. Ficek and R. Tanas, Phys. Rep. 372, 369 (2002).

[4] D. Leibfried, R. Blatt, C. Monroe, and D. Wineland, Rev. Mod. Phys. 75, 281 (2003).

[5] I. Bloch, Nature (London) 453, 1016 (2008).

[6] R.G. De Voe and R.G. Brewer, Phys. Rev. Lett. 76, 2049 (1996).

[7] J.M. Raimond, P. Goy, M. Gross, C. Fabre, and S. Haroche, Phys. Rev. Lett. 49, 1924 (1982).

[8] G. R. Guthöhrlein, M. Keller, K. Hayasaka, W. Lange, and H. Walther, Nature (London) 414, 49 (2001); M. Keller, B. Lange, K. Hayasaka, W. Lange, and H. Walther, Nature (London) 431, 1075 (2004).

[9] A. B. Mundt, A. Kreuter, C. Becher, D. Leibfried, J. Eschner, F. Schmidt-Kaler, and R. Blatt, Phys. Rev. Lett. 89, 103001 (2002); A. Kreuter, C. Becher, G. P. T. Lancaster, A. B. Mundt, C. Russo, H. Häffner, C. Roos, J. Eschner, F. Schmidt-Kaler, and R. Blatt, Phys. Rev. Lett. 92, 203002 (2004).

[10] G. Benivegna and A. Messina, J. Mod. Opt. 36, 1205 (1989).

[11] G. Benivegna and A. Messina, Phys. Lett. A 126, 249 (1988).

[12] V. Bužek, Z. Phys. D 17, 91 (1990).

[13] P. F. Herskind, A. Dantan, J. P Marler, M. Albert, and M. Drewsen, Nature Phys. 5, 494 (2009).

[14] M. Tavis and F. W. Cummings, Phys. Rev. 170, 379 (1968).

[15] H. J. Kimble, Nature (London) 453, 1023 (2008).
[16] T. Pellizzari, S. A. Gardiner, J. I. Cirac, and P. Zoller, Phys. Rev. Lett. 75, 3788 (1995).

[17] S. J. van Enk, J. I. Cirac, and P. Zoller, Phys. Rev. Lett. 79, 5178 (1997).

[18] M. B. Plenio, S. F. Huelga, A. Beige, and P. L. Knight, Phys. Rev. A 59, 2468 (1999).

[19] S.-B. Zheng and G.-C. Guo, Phys. Rev. Lett. 85, 2392 (2000).

[20] J. Pachos and H. Walther, Phys. Rev. Lett. 89, 187903 (2002).

[21] P. Lougovski, E. Solano, and H. Walther, Phys. Rev. A 71, 013811 (2005).

[22] G. Chimczak, R. Tanaś, and A. Miranowicz, Phys. Rev. A 71, 032316 (2005).

[23] S.-B. Li and J.-B. Xu, Phys. Rev. A 72, 022332 (2005).

[24] S.-B. Li, Phys. Rev. A 75, 054304 (2007).

[25] G. Chimczak and R. Tanaś, Phys. Rev. A 77, 032312 (2008).

[26] M. Bina, F. Casagrande, A. Lulli, and E. Solano, Phys. Rev. A 77, 033839 (2008).

[27] S. Natali and Z. Ficek, Phys. Rev. A 75, 042307 (2007).

[28] L. Mazzola, S. Maniscalco, J. Piilo, K.-A. Suominen, and B. M. Garraway, Phys. Rev. A 79, 042302 (2009).

[29] S. Maniscalco, F. Francica, R. L. Zaffino, N. Lo Gullo, and F. Plastina, Phys. Rev. Lett. 100, 090503 (2008).

[30] M. Keller, B. Lange, K. Hayasaka, W. Lange, and H. Walther, New J. Phys. 6, 95 (2004).

[31] T. Monz, K. Kim, W. Hänsel, M. Riebe, A. S. Villar, P. Schindler, M. Chwalla, M. Hennrich, and R. Blatt, Phys. Rev. Lett. 102, 040501 (2009). 
[32] C. Di Fidio, S. Maniscalco, W. Vogel and A. Messina, Phys. Rev. A 65, 033825 (2002).

[33] J. Dalibard, Y. Castin, and K. Mølmer, Phys. Rev. Lett. 68, 580 (1992).

[34] R. Dum, P. Zoller, and H. Ritsch, Phys. Rev. A 45, 4879 (1992); R. Dum, A. S. Parkins, P. Zoller, and C. W. Gardiner, Phys. Rev. A 46, 4382 (1992); H. Carmichael, An Open System Approach to Quantum Optics, Lecture
Notes in Physics (Springer-Verlag, Berlin, 1993), Vol. $\mathrm{m} 18$.

[35] W. K. Wootters, Phys. Rev. Lett. 80, 2245 (1998).

[36] W. Lange (private communication).

[37] F. Francica, S. Maniscalco, J. Piilo, F. Plastina, and K.A. Suominen, Phys. Rev. A 79, 032310 (2009). 